\title{
Delay-aware BS Discontinuous Transmission Control and User Scheduling for Energy Harvesting Downlink Coordinated MIMO Systems
}

\author{
Ying Cui, MIEEE, Vincent K. N. Lau, FIEEE, Yueping Wu, StMIEEE \\ ECE Department, Hong Kong University of Science and Technology, Hong Kong \\ Email: cuiying@ust.hk, eeknlau@ece.ust.hk, eewyp@ust.hk
}

\begin{abstract}
In this paper, we propose a two-timescale delay-optimal base station Discontinuous Transmission (BS-DTX) control and user scheduling for downlink coordinated MIMO systems with energy harvesting capability. To reduce the complexity and signaling overhead in practical systems, the BS-DTX control is adaptive to both the energy state information (ESI) and the data queue state information (QSI) over a longer timescale. The user scheduling is adaptive to the ESI, the QSI and the channel state information (CSI) over a shorter timescale. We show that the two-timescale delay-optimal control problem can be modeled as an infinite horizon average cost Partially Observed Markov Decision Problem (POMDP), which is well-known to be a difficult problem in general. By using sample-path analysis and exploiting specific problem structure, we first obtain some structural results on the optimal control policy and derive an equivalent Bellman equation with reduced state space. To reduce the complexity and facilitate distributed implementation, we obtain a delay-aware distributed solution with the BS-DTX control at the BS controller (BSC) and the user scheduling at each cluster manager (CM) using approximate dynamic programming and distributed stochastic learning. We show that the proposed distributed two-timescale algorithm converges almost surely. Furthermore, using queueing theory, stochastic geometry and optimization techniques, we derive sufficient conditions for the data queues to be stable in the coordinated MIMO network and discuss various design insights. Finally, we compare the proposed algorithm with various baseline schemes and show that significant delay performance gain can be achieved.
\end{abstract}

\section{Index Terms}

delay-aware, base station discontinuous transmission control (BS-DTX), interference network, renewable energy, energy harvesting system, distributed stochastic learning, queueing theory, stochastic geometry. 


\section{INTRODUCTION}

Inter-cell interference is a critical performance bottleneck in cellular networks. The interference mitigation techniques can be roughly classified into two types, namely coordinated MIMO techniques and cooperative MIMO techniques, according to the required backhaul consumption [1]. For coordinated MIMO techniques, only the channel state information (CSI) is shared among MIMO base stations (BSs) through backhaul for the coordinated beamforming design at each BS to combat interference [2]. On the other hand, for cooperative MIMO techniques, both the CSI and the payload data are shared among MIMO BSs through backhaul for joint precoder designs at all the BSs to combat interference [3]. Since CSI sharing is performed for each transmission frame, while data sharing is operated for each data symbol, coordinated MIMO consumes much less backhaul capacity than cooperative MIMO at the expense of performance (e.g., degrees of freedom).

Due to the limited degrees of freedom and the limited backhaul capacity at each BS, global cooperation or coordination of all the BSs in the network is not possible and the BSs are organized into disjoint clusters [3]-[7]. The BSs within each cluster cooperatively serve the users associated with them, which lowers the system complexity and completely eliminates intra-cluster interference. For example, in [4], multi-antenna BSs in each fixed cluster adopt coordinated beamforming to serve the single-antenna users in their own cells and avoid the interference to the users served by other BSs in the same cluster. In [3], [5], the authors propose a BS cooperation strategy for fixed clusters, including full intra-cluster cooperation to eliminate intra-cluster interference and limited inter-cluster coordination to reduce the interference for the cluster edge users based on the per-cluster CSI and the CSI of the edge users in the neighboring clusters. In [6], [7], the authors consider different types of static cluster-based cooperation schemes in a multi-cell system with multiple sectors per cell.

However, all these works focus on physical layer performance (such as sum throughput, transport capacity) in cellular networks. They ignore the bursty data arrivals and assume infinite backlogs of packets at the transmitter. In other words, the information flows are assumed to be delay insensitive. The resulting control policy is adaptive to the CSI only and it cannot guarantee good delay performance for delay-sensitive applications [3]-[7]. In practice, a lot of applications have bursty arrivals and they are delay-sensitive. It is very important to take into account the delay performance in designing the cross-layer interference control algorithms for the coordinated MIMO systems. The control policy for delay-sensitive applications should be adaptive to both the CSI and the queue state information 1 (QSI). The motivation can be illustrated by the following example, as illustrated in Fig. 11(a). Under cluster-based cooperative or coordinated MIMO, MSs only suffer from inter-cluster interference, as

\footnotetext{
${ }^{1}$ The CSI gives the knowledge about good opportunity to transmit whereas the QSI gives the knowledge about the urgency of the data flow.
} 
intra-cluster interference is eliminated. Therefore, cluster edge MSs suffer much more interference than cluster center MSs. In this work, we are interested to investigating delay-aware BS-discontinuous transmission (BS-DTX) control and user scheduling to reduce inter-cluster interference and save energy of the whole network. To maximize the sum throughput, the CSI-based BS-DTX control and user scheduling always favors cluster center MSs while starves cluster edge MSs. This may lead to infinite delay of cluster edge MSs and hence, infinite average delay of all the MSs. However, the QSI and CSI based design will dynamically favor different types of MSs to capture the urgency of data flows and the good opportunity of channels. Therefore, it can guarantee good delay performance. However, the design framework taking into account the queueing delay and the physical layer performance is far from trivial as it involves both queuing theory (to model the queuing dynamics) and information theory (to model the physical layer dynamics).

In addition, recent initiatives towards green communications have driven the design of wireless infrastructure to be more energy-efficient. One energy-efficient design is to exploit renewable energy at BSs. There are many recent works on power management in energy harvesting networks. For example, in [8], [9], the authors extend the Lyapunov optimization framework to derive an efficient energy management algorithm for energy harvesting networks. In [10], the authors consider dynamic node activation in energy harvesting sensor networks and propose a simple threshold-based node activation policy to achieve near-optimal system throughput. Similarly, all these papers have focused on physical layer throughput performance and the nodes are powered by renewable energy source only with infinite energy storage size.

In this paper, we consider delay-optimal BS-DTX control and user scheduling algorithm in downlink energy harvesting coordinated MIMO systems with limited renewable energy storage. Each BS is powered by both conventional grid and renewable power sources. There are various first-order technical challenges involved in solving the problem.

- Renewable and Grid Power Control: The transmit power of a BS comes from both renewable and grid power sources, which have very different properties. For instance, the grid power has stable power supply but there is cost associated with it. On the other hand, the renewable power is virtually free but it has random supply and hence, an energy storage is needed for efficient utilization of the renewable energy. In practice, the energy storage has limited capacity and hence, the BS power control and user scheduling algorithm should be adaptive to the renewable energy state information (ESI) and the data QSI as well as the CSI. It is highly nontrivial to strike a balance between these factors in the control algorithm design.

- Delay-aware Low Complexity Distributed Algorithm: While the delay-optimal control problem can be casted into an Markov Decision Process (MDP), brute force solutions such as value iteration and policy iteration will suffer from the curse of dimensionality [11]. For example, a very large 
state space (exponential to the number of users in the network) will be involved. In addition to the complexity issue, the solution obtained will be centralized and it requires knowledge of global system state information (ESI, QSI, CSI). However, these system state information is usually distributed locally at various BSs and huge signaling overhead will be involved in collecting these information. Therefore, it is highly desirable to obtain a delay-aware low complexity and distributed algorithm with guaranteed delay performance.

- Performance Analysis: Besides algorithm development, it is important to analyze the system performance to understand how it is affected by the renewable energy storage size and the interference coupling in cellular networks. One challenge on the system performance analysis is the statistical characterization of interference. In [12], the authors study the coverage and rate of cellular networks without BS coordination using stochastic geometry [13]. The locations of the BSs are modeled as a homogeneous Poisson point process (PPP) and the locations of the mobile stations (MSs) are modeled as some independent (of the point process of BSs) point process. The analysis for coordinated MIMO network is more challenging due to the asymmetric topology induced by clustering. In addition, the analysis becomes more involved when queueing dynamics of data queues and renewable energy queues are considered.

In this paper, considering the limited backhaul capacity and the latency in information exchange through backhaul in practical cellular systems [7], we adopt cluster-based coordinated2 MIMO to eliminate intra-cluster interference. We propose a two-timescale delay-aware BS-DTX control and user scheduling for energy harvesting downlink coordinated MIMO systems as illustrated in Fig. 1 (a). The BS-DTX control is adaptive to both the ESI and the QSI over a longer timescale. The user scheduling is adaptive to the ESI, the QSI and the CSI over a shorter timescale. We show that the two-timescale delay-optimal control problem can be modeled as an infinite horizon average cost Partially Observed Markov Decision Process (POMDP), which is well-known to be a difficult problem [14]. By using sample-path analysis and exploiting the specific problem structure, we first obtain some structural results on the optimal control policy and derive an equivalent Bellman equation with reduced state space. To derive a distributed control policy, we approximate the Q-factor and potential function associated with the equivalent Bellman equation by the per-flow functions. The per-flow functions are estimated online using distributed stochastic learning at each BS. We prove the almost-sure convergence of the proposed distributed algorithm. Furthermore, using queueing theory, stochastic geometry and optimization techniques, we characterize the sufficient conditions for data queues in the coordinated MIMO networks to be stable. Based on the analysis, we discuss the impacts of the

\footnotetext{
${ }^{2}$ The design framework proposed in this paper does not rely on specific physical layer transmission schemes and can be easily extended to cluster-based cooperative MIMO.
} 
interference coupling and the size of renewable energy storage on network performance. Finally, we compare the proposed algorithm with various baseline schemes and show that significant delay performance gain can be achieved.

\section{System Models}

In this section, we shall elaborate on the system architecture, the physical layer model as well as the bursty source model for the coordinated MIMO networks.

\section{A. Architecture of Downlink Distributed MIMO Systems}

We consider a downlink coordinated MIMO system consisting of $B$ multi-antenna BSs and $K$ single-antenna MSs as illustrated in Fig. 1 (a). Each BS has $N_{t}$ transmit antennas. Let $\mathcal{K}_{b}$ denote the set of $K_{b}$ MS indices associated with the $b$-th BS and $\mathcal{K}$ denote the set of $K=\sum_{b} K_{b}$ MS indices in the network. The set of BSs $\mathcal{B}=\{1, \cdots, B\}$ are partitioned into $N=B / N_{t}$ coordination cluster 3 , i.e., $\mathcal{B}=\cup_{n=1}^{N} \mathcal{B}_{n}$ and $\mathcal{B}_{n} \cap \mathcal{B}_{n^{\prime}}=\emptyset \forall n \neq n^{\prime}$, where $\mathcal{B}_{n}$ denotes the set of $B_{n}$ BSs in cluster $n$. Each coordination cluster contains $N_{t}$ neighboring BSs and is managed by a cluster manager (CM) and all the $N$ CMs are managed by a BS controller (BSC). The BSs in the same cluster share the CSI and perform coordinated beamforming [1] to combat intra-cluster interference. Besides conventional grid power source, each BS is able to harvest energy from the environment, e.g., using solar panels [15]. At each BS, there is a renewable energy queue (battery) with limited capacity for storing the harvested energy. In addition, at each BS, there are multiple data queues for buffering the packets to all the MSs associated with the BS (one queue for each MS) as illustrated in Fig. 11 (a).

\section{B. Physical Layer Model}

Let $\mathbf{h}_{k, b} \in \mathcal{H}$ and $L_{k, b}$ denote the $N_{t} \times 1$ complex small-scale fading vector and the long-term path gain between the $b$-th BS and the $k$-th MS, where $\mathcal{H} \subset \mathbb{C}^{N_{t} \times 1}$ denotes the finite discrete complex CSI state space. Let $\mathbf{H}_{n}=\left\{\mathbf{h}_{k, b}: k \in \mathcal{K}_{b}, b \in \mathcal{B}_{n}\right\} \in \mathcal{H}_{n} \triangleq \mathcal{H}^{\sum_{b \in \mathcal{B}_{n}} K_{b}}$ and $\mathbf{H}=\cup_{n=1}^{N} \mathbf{H}_{n} \in \mathcal{H} \triangleq \mathcal{H}^{K}$ denote the intra-cluster CSI at $n$-th CM and the aggregation of the CSI over $N$ clusters, respectively. In this paper, the time dimension is partitioned into scheduling slots indexed by $t$ with slot duration $\tau$ (second).

Assumption 1 (Quasi-static Fading): $\mathbf{h}_{k, b}(t)$ is quasi-static in each scheduling slot for all $(k, b) \in$ $\mathcal{K} \times \mathcal{B}$. Furthermore, each element of vector $\mathbf{h}_{k, b}(t)$ follows a general distribution with mean 0 and vairiance 1 . The distribution of each element of vector $\mathbf{h}_{k, b}(t)$ is i.i.d. over scheduling slots and independent w.r.t. $\{k, b\}$. The long-term path gain $L_{k, b}$ remains constant for the duration of the communication session.

\footnotetext{
${ }^{3}$ For simplicity, we assume $B$ is a multiple of $N_{t}$.
} 
We assume all the BSs in the system share a common spectrum. Let $p_{b} \in \mathcal{P} \triangleq\{0,1\}$ denote the binary BS-DTX control action of the $b$-th BS, where $p_{b}=1$ indicates the $b$-th $\mathrm{BS}$ is active and $p_{b}=0$ otherwise. Between the coordination clusters, the inter-cluster interference is managed by a binary BS-DTX control action $\mathbf{p}=\left\{p_{b}: p_{b} \in \mathcal{P}, b \in \mathcal{B}\right\} \in \mathcal{P}$, where $\mathcal{P} \subseteq \mathcal{P}^{B}$ is the aggregate BS-DTX control action space and specifies the BS-DTX patterns [16]. Since each BS has renewable and grid power sources, we have $p_{b}=p_{b}^{E}+p_{b}^{G}$, where $p_{b}^{E} \in \mathcal{P}$ and $p_{b}^{G} \in \mathcal{P}$ denote the power contribution from the renewable power and grid power sources of the $b$-th BS, respectively. Let $s_{k} \in \mathcal{S} \triangleq\{0,1\}$ denote the user scheduling action of the $k$-th MS, where $s_{k}=1$ indicates the $k$-th MS is selected to receive packets and $s_{k}=0$ otherwise. Thus, users are selected according to a user scheduling action $\mathbf{s}=\left\{s_{k}: s_{k} \in \mathcal{S}, k \in \mathcal{K}\right\} \in \mathcal{S}$, where $\mathcal{S} \subseteq \mathcal{S}^{K}$ is the aggregate user scheduling action space. The BS-DTX control and user scheduling are performed according to a control policy to be defined in Definition 1

In each slot, each active BS selects one MS to serve. Within each coordination cluster, the active BSs combat the intra-cluster interference using coordinated beamforming [1], [2], [4]. Let $P_{b}$ and $x_{k}$ denote the instantaneous transmit power of the $b$-th BS and the information symbols for the $k$-th MS, respectively. The received signal at the $k$-th MS of the $b$-th cell in the $n$-th cluster is given by

$$
\begin{aligned}
y_{k}= & \underbrace{p_{b} \sqrt{P_{b}} \sqrt{L_{k, b}} \mathbf{h}_{k, b}^{T} \mathbf{w}_{k, b} s_{k} x_{k}}_{\text {desired signal }}+\underbrace{\sum_{b^{\prime} \in \mathcal{B}_{n}, b^{\prime} \neq b} p_{b^{\prime}} \sqrt{P_{b^{\prime}}} \sqrt{L_{k, b^{\prime}}} \mathbf{h}_{k, b^{\prime}}^{T}\left(\sum_{k^{\prime} \in \mathcal{K}_{b^{\prime}}} \mathbf{w}_{k^{\prime}, b^{\prime}} s_{k^{\prime}} x_{k^{\prime}}\right)}_{\text {intra-cluster interference }} \\
& +\underbrace{\sum_{n^{\prime} \neq n} \sum_{b^{\prime} \in \mathcal{B}_{n^{\prime}}} p_{b^{\prime}} \sqrt{P_{b^{\prime}}} \sqrt{L_{k, b^{\prime}}} \mathbf{h}_{k, b^{\prime}}^{T}\left(\sum_{k^{\prime} \in \mathcal{K}_{b^{\prime}}} \mathbf{w}_{k^{\prime}, b^{\prime}} s_{k^{\prime}} x_{k^{\prime}}\right)}_{\text {inter-cluster interference }}+\underbrace{z_{k}}_{\text {noise }}, k \in \mathcal{K}_{b}, b \in \mathcal{B}_{n}
\end{aligned}
$$

where $z_{k} \sim \mathcal{C N}\left(0, N_{0}\right)$ is the AWGN noise and $\mathbf{w}_{k, b} \in \mathbb{C}^{N_{t} \times 1}$ is the zero-forcing beamforming weight for the $k$-th MS at the $b$-th BS. Specifically, $\left\{\mathbf{w}_{k, b}\right\}$ is given by the solution 4 of the zeroforcing problem: $\sum_{k \in \mathcal{K}_{b}}\left\|\mathbf{w}_{k, b}\right\|^{2} s_{k}=p_{b}$ and $s_{k} \mathbf{h}_{k, b^{\prime}}^{T}\left(\sum_{k^{\prime} \in \mathcal{K}_{b^{\prime}}} \mathbf{w}_{k^{\prime}, b^{\prime}} s_{k^{\prime}}\right)=0\left(\forall b^{\prime} \in \mathcal{B}_{n}, b^{\prime} \neq b\right)$.

The receive SINR at the $k$-th MS of the $b$-th cell in the $n$-th cluster is given by

$$
\rho_{k}(\mathbf{H}, \mathbf{p}, \mathbf{s})=\frac{P_{k}^{r x}}{N_{0}+I_{k}}, k \in \mathcal{K}_{b}, b \in \mathcal{B}_{n}
$$

where the receive power $P_{k}^{r x}$ and the inter-cluster interference power $I_{k}$ are given by

$$
\begin{gathered}
P_{k}^{r x}=p_{b} P_{b} L_{k, b}\left\|\mathbf{h}_{k, b}^{T} \mathbf{w}_{k, b}\right\|^{2} s_{k} \\
I_{k}=\sum_{n^{\prime} \neq n} \sum_{b^{\prime} \in \mathcal{B}_{n^{\prime}}} p_{b^{\prime}} P_{b^{\prime}} L_{k, b^{\prime}}\left\|\mathbf{h}_{k, b^{\prime}}^{T}\left(\sum_{k^{\prime} \in \mathcal{K}_{b^{\prime}}} \mathbf{w}_{k^{\prime}, b^{\prime}} s_{k^{\prime}}\right)\right\|^{2}
\end{gathered}
$$

We have the following assumption regarding packet transmission.

\footnotetext{
${ }^{4}$ If there are more than one solutions, we choose the one maximizes $\left\|\mathbf{h}_{k, b}^{T} \mathbf{w}_{k, b}\right\|^{2}$.
} 
Assumption 2 (Packet Transmission Model): One data packet with certain fixed packet size can be successfully received by the $k$-th MS if the receive SINR $\rho_{k}$ exceeds a certain threshold $5 \delta_{k}$, i.e., $\rho_{k} \geq \delta_{k}$. There exists a state-action pair $(\mathbf{H}, \mathbf{p}, \mathbf{s}) \in \mathcal{H} \times \mathcal{P} \times \mathcal{S}$, such that $\operatorname{Pr}\left[\rho_{k}(\mathbf{H}, \mathbf{p}, \mathbf{s}) \geq \delta_{k}\right]>0$.

\section{Bursty Source Model and Queue Dynamics}

Let $\mathbf{A}^{Q}(t)=\left\{A_{k}^{Q}(t): k \in \mathcal{K}\right\}$ and $\mathbf{A}^{E}(t)=\left\{A_{b}^{E}(t): b \in \mathcal{B}\right\}$ be the number of packets arriving to the $K \mathrm{MSs}$ and the number of renewable energy units 6 arriving to the $B$ BSs at the end of the $t$-th scheduling slot, respectively. We have the following assumptions] regarding the bursty data and renewable energy arrival processes.

Assumption 3 (Bursty Data Source Model): The arrival process $A_{k}^{Q}(t)$ is i.i.d. over scheduling slots and independent w.r.t. $k$ according to a general distribution $P_{A_{k}^{Q}}(\cdot)$ with average arrival rate $\mathbb{E}\left[A_{k}^{Q}(t)\right]=\lambda_{k}^{Q}<1$. The statistics of $A_{k}^{Q}(t)$ is unknown to the controller.

Assumption 4 (Bursty Renewable Energy Model): The arrival process $A_{b}^{E}(t)$ is i.i.d. over scheduling slots and independent w.r.t. $b$ according to a general distribution $P_{A_{b}^{E}}(\cdot)$ with average arrival rate $\mathbb{E}\left[A_{b}^{E}(t)\right]=\lambda_{b}^{E}<1$. The statistics of $A_{b}^{E}(t)$ is unknown to the controller.

Remark 1 (Interpretation of Assumption 4): Assumption 44implies that the renewable power source is stationary. Although the renewable energy source is not stationary over a very long time horizon in practice, it is stationary over a typical communication session, which lasts for less than 30 mins.

Let $\mathbf{Q}_{n}(t)=\left\{Q_{k}(t): k \in \mathcal{K}_{n}\right\} \in \mathcal{Q}_{n} \triangleq \mathcal{Q}^{\sum_{b \in \mathcal{B}_{n}} K_{b}}$ be the $n$-th cluster QSI and $\mathbf{Q}(t)=$ $\cup_{n=1}^{N} \mathbf{Q}_{n}(t) \in \mathcal{Q} \triangleq \mathcal{Q}^{K}$ be the aggregation of the QSI over $N$ clusters at the beginning of the $t$-th slot, where $Q_{k}(t) \in \mathcal{Q} \triangleq\left\{0,1, \cdots, N_{Q}\right\}$ denotes the number of data packets at the data queue for the $k$-th MS and $N_{Q}$ denotes the data buffer size. At slot $t$, there is $\mathbf{I}\left[\rho_{k}(t) \geq \delta_{k}\right] \in\{0,1\}$ packet successfully received at the $k$-th MS, where $\mathbf{I}[\cdot]$ denotes the indicator function. Hence, the data queue dynamics of the $k$-th MS is given by

$$
Q_{k}(t+1)=\min \left\{\left[Q_{k}(t)-\mathbf{I}\left[\rho_{k}(t) \geq \delta_{k}\right]\right]^{+}+A_{k}^{Q}(t), N_{Q}\right\}, k \in \mathcal{K}
$$

\footnotetext{
${ }^{5}$ In general, we allow different MSs with different packet sizes, and hence the threshold is indexed by $k$ and may be different for different MSs.

${ }^{6}$ One unit of energy for the $b$-th BS corresponds to the amount of energy consumed in downlink transmission at each slot for the $b$-th BS, i.e. $P_{b} \tau$ Joule. Note that the instantaneous transmit power from the renewable power source is finite (i.e., $P_{b}$ ). The notion "unit of energy" can be easily extended from binary (on-off) power control to handle (multi-level) power control.

${ }^{7}$ Note that under Assumption 3 and Assumption 4, we have $\operatorname{Pr}\left[A_{k}^{Q}(t)=0\right]>0$ and $\operatorname{Pr}\left[A_{b}^{E}(t)=0\right]>0$ for all $k \in \mathcal{K}$ and $b \in \mathcal{B}$, respectively.
} 
where $\rho_{k}(t) \triangleq(\mathbf{H}(t), \mathbf{p}(t), \mathbf{s}(t))$ and $x^{+} \triangleq \max \{x, 0\}$.

Similarly, let $\mathbf{E}_{n}(t)=\left\{E_{b}(t): b \in \mathcal{B}_{n}\right\} \in \mathcal{E}_{n} \triangleq \mathcal{E}^{B_{n}}$ be the $n$-th cluster ESI and $\mathbf{E}(t)=$ $\cup_{n=1}^{N} \mathbf{E}_{n}(t) \in \mathcal{E} \triangleq \mathcal{E}^{B}$ be the aggregation of the ESI over $N$ clusters at the beginning of the $t$-th slot, where $E_{b}(t) \in \mathcal{E} \triangleq\left\{0,1, \cdots, N_{E}\right\}$ denotes the number of renewable energy units in the energy queue for the $b$-th BS and $N_{E}$ denotes the energy storage size. At slot $t, p_{b}^{E}(t) \in \mathcal{P}$ unit of renewable energy is consumed from the $b$-th energy queue for packet transmission. Hence, the energy queue dynamics of the $b$-th $\mathrm{BS}$ is given by

$$
E_{b}(t+1)=\min \left\{\left[E_{b}(t)-p_{b}^{E}(t)\right]^{+}+A_{k}^{E}(t), N_{E}\right\}, b \in \mathcal{B}
$$

\section{BS-DTX Control and User Scheduling Policy}

For notation convenience, we denote $\boldsymbol{\chi}(t)=(\mathbf{E}(t), \mathbf{Q}(t), \mathbf{H}(t)) \in \mathcal{X}=\mathcal{E} \times \mathcal{Q} \times \mathcal{H}$ as the global system state at the $t$-th slot. We first define the centralized control policy. Specifically, at the beginning of each slot, the controller determines the renewable power DTX control action $\mathbf{p}^{E}=\left\{p_{b}^{E}: p_{b}^{E} \in\right.$ $\mathcal{P}, b \in \mathcal{B}\} \in \mathcal{P}$, grid power DTX control action $\mathbf{p}^{G}=\left\{p_{b}^{G}: p_{b}^{G} \in \mathcal{P}, b \in \mathcal{B}\right\} \in \mathcal{P}$ as well as the user scheduling action $\mathbf{s}=\left\{s_{k}: s_{k} \in \mathcal{S}, k \in \mathcal{K}\right\} \in \mathcal{S}$ based on the global system state $\chi(t)$ according to the control policy defined below.

Definition 1 (BS-DTX Control and User Scheduling Policy): A BS-DTX control and user scheduling policy consists of a sequence of mappings $\pi=\left\{\Omega^{1}, \Omega^{2}, \cdots\right\}$. The mapping for the $t$-th slot $\Omega^{t}=\left(\Omega_{p}^{E, t}, \Omega_{p}^{G, t}, \Omega_{s}^{t}\right)$ is a mapping from the system state $\chi(t) \in \mathcal{X}$ to the renewable power DTX control action $\Omega_{p}^{E, t}(\mathbf{E}(t), \mathbf{Q}(t))=\mathbf{p}^{E}(t) \in \mathcal{P}$, the grid power DTX control action $\Omega_{p}^{G, t}(\mathbf{E}(t), \mathbf{Q}(t))=$ $\mathbf{p}^{G}(t) \in \mathcal{P}$ and the user scheduling action $\Omega_{s}^{t}(\chi(t))=\mathbf{s}(t) \in \mathcal{S}$. A policy $\pi$ is called feasible if for all $t$, the following constraints are satisfied:

1) $p_{b}^{E}(t)=0$ if $E_{b}(t)=0$ for all $b \in \mathcal{B}$ (no renewable energy available for transmission).

2) $p_{b}(t)=p_{b}^{E}(t)+p_{b}^{G}(t) \in \mathcal{P}$ for all $b \in \mathcal{B}$ (binary BS-DTX control).

3) $\sum_{k \in \mathcal{K}_{b}} s_{k}(t)=p_{b}(t)$ for all $b \in \mathcal{B}$ (each active BS selects one MS in its cell).

Remark 2 (Motivation of Two-Timescale Control Policy): The two-timescale control is a constraint we impose due to the following practical reasons. The QSI and ESI are changing on a longer timescale (e.g., several slots) while the CSI is changing on a shorter timescale (e.g., per-slot). The BS-DTX control is usually implemented at the BSC for interference reduction and energy saving of the whole network. As a result, the BS-DTX control cannot afford to be running on a per-slot basis, due to the high complexity and signaling overhead in collecting the local CSI from all the BSs. Therefore, it is desirable to make it a function of the ESI and QSI only. On the other hand, the low complexity distributed user scheduling is implemented locally at each CM (similar to HSDPA in current 3G networks) and they can afford to run on a per-slot basis and adapt to the ESI, QSI and CSI. 


\section{Problem Formulation AND Optimal SOlution}

In this section, we shall first elaborate on the dynamics of the system state under a control policy $\pi$. Based on that, we shall formulate the delay-optimal control problem and derive some structural properties for the optimal solution.

\section{A. Delay-Optimal Problem Formulation}

Under Assumptions 1, 3 and 4 the induced random process $\{\chi(t)\}$ for a given feasible control policy $\pi=\left\{\Omega^{1}, \Omega^{2}, \cdots\right\}$ is a Markov chain with the following transition probability

$$
\begin{aligned}
& \operatorname{Pr}\left[\boldsymbol{\chi}(t+1) \mid \boldsymbol{\chi}(t), \Omega^{t}(\boldsymbol{\chi}(t))\right] \\
= & \operatorname{Pr}\left[\mathbf{H}(t+1) \mid \boldsymbol{\chi}(t), \Omega^{t}(\boldsymbol{\chi}(t))\right] \operatorname{Pr}\left[\mathbf{E}(t+1) \mid \boldsymbol{\chi}(t), \Omega^{t}(\boldsymbol{\chi}(t))\right] \operatorname{Pr}\left[\mathbf{Q}(t+1) \mid \chi(t), \Omega^{t}(\boldsymbol{\chi}(t))\right] \\
= & \operatorname{Pr}[\mathbf{H}(t+1)] \operatorname{Pr}\left[\mathbf{E}(t+1) \mid \chi(t), \Omega^{t}(\boldsymbol{\chi}(t))\right] \operatorname{Pr}\left[\mathbf{Q}(t+1) \mid \boldsymbol{\chi}(t), \Omega^{t}(\boldsymbol{\chi}(t))\right]
\end{aligned}
$$

As a result, given a feasible control policy $\pi$, the average delay cost per stage of the $k$-th MS starting from a given initial state $\chi(1)$ is given by

$$
\overline{D_{\pi, k}}(\chi(1))=\limsup _{T \rightarrow \infty} \frac{1}{T} \mathbb{E}^{\pi}\left[\sum_{t=1}^{T} f\left(Q_{k}(t)\right)\right], \forall k \in \mathcal{K}
$$

where the expectation is taken w.r.t. the measure induced by the policy $\pi$ and $f\left(Q_{k}\right)$ is a monotonic increasing utility function of $Q_{k}$. For example, with $f\left(Q_{k}\right)=\frac{Q_{k}}{\lambda_{k}}$ and $f\left(Q_{k}\right)=\mathbf{1}\left[Q_{k} \geq Q_{k}^{o}\right]\left(Q_{k}^{o} \in\right.$ $\left.\left\{0, \cdots, N_{Q}\right\}\right),(7)$ can be used to measure the average delay and the average queue outage probability of the $k$-th MS under policy $\pi$. Similarly, given a feasible control policy $\pi$, the average grid power cost per stage of the $b$-th BS starting from a given initial state $\chi(1)$ is given by

$$
\overline{p_{\pi, b}^{G}}(\chi(1))=\limsup _{T \rightarrow \infty} \frac{1}{T} \mathbb{E}^{\pi}\left[\sum_{t=1}^{T} p_{b}^{G}(t)\right], \forall b \in \mathcal{B}
$$

We are interested in minimizing the average delay cost of each MS $k \in \mathcal{K}$ in (7) and the average grid power cost of each BS $b \in \mathcal{B}$ in (8). A Pareto optimal tradeoff on the average delay and average grid power consumption can be obtained by solving the following problem.

Problem 1 (Two-Timescale Delay-Optimal Control): For some positive constants $\boldsymbol{\beta}=\left\{\beta_{k}>0\right.$ : $k \in \mathcal{K}\}$ and $\gamma=\left\{\gamma_{b}>0: b \in \mathcal{B}\right\}$, the delay-optimal problem is formulated as

$$
\min _{\pi} J_{\pi}^{(\boldsymbol{\beta}, \boldsymbol{\gamma})}(\chi(1))=\sum_{k \in \mathcal{K}} \beta_{k} \overline{D_{\pi, k}}(\chi(1))+\sum_{b \in \mathcal{B}} \gamma_{b} \overline{p_{\pi, b}^{G}}(\chi(1))=\lim _{T \rightarrow \infty} \frac{1}{T} \mathbb{E}^{\pi}\left[\sum_{t=1}^{T} g\left(\chi(t), \Omega^{t}(\chi(t))\right)\right]
$$

where $g\left(\chi(t), \Omega^{t}(\chi(t))\right)=\sum_{k \in \mathcal{K}} \beta_{k} f\left(Q_{k}(t)\right)+\sum_{b \in \mathcal{B}} \gamma_{b} p_{b}^{G}(t)$ and the control policy $\pi$ satisfies the two-timescale requirement in Definition 1

Remark 3 (Two-Timescale Control and POMDP): By two-timescale requirement, the BS-DTX control policy is defined on the partial system state $(\mathbf{E}, \mathbf{Q})$, while the user scheduling policy is defined on 
the complete system state $\chi=(\mathbf{E}, \mathbf{Q}, \mathbf{H})$. Due to the two-timescale control constraint as in Definition 1. Problem 1 is a POMDP 8 .

\section{B. Policy and State Space Reduction}

Problem 1 belongs to POMDP, which is well-known to be a challenging problem in general. Yet, we shall exploit some special structures in our problems to reduce the policy and state spaces. Based on that, we can simplify the POMDP. We first have the following lemma on the structural property of the BS-DTX control, which helps to reduce the policy space.

Lemma 1 (Structure of Optimal BS-DTX Control): Let the BS-DTX control for the $t$-th slot be denoted by $\Omega_{p}^{t}: \mathcal{E} \times \mathcal{Q} \rightarrow \mathcal{P}$, which is a mapping from the partial system state $(\mathbf{E}, \mathbf{Q}) \in \mathcal{E} \times \mathcal{Q}$ to the BS-DTX control action $\Omega_{p}^{t}(\mathbf{E}(t), \mathbf{Q}(t))=\mathbf{p}(t) \in \mathcal{P}$. Conditioned on any $\Omega_{p, b}^{t}$, the optimal $\Omega_{p, b}^{E, t}$ and $\Omega_{p, b}^{G, t}$ satisfy $\Omega_{p, b}^{E, t}(\mathbf{E}(t), \mathbf{Q}(t))=\Omega_{p, b}^{t}(\mathbf{E}(t), \mathbf{Q}(t)) \mathbf{I}\left[E_{b}(t)>0\right]$ and $\Omega_{p, b}^{G, t}(\mathbf{E}(t), \mathbf{Q}(t))=$ $\Omega_{p, b}^{t}(\mathbf{E}(t), \mathbf{Q}(t)) \mathbf{I}\left[E_{b}(t)=0\right]$ for all $b \in \mathcal{B}$ and all $t$.

Proof: Please refer to Appendix A.

Remark 4 (Interpretation of Lemma \): Lemma 1 indicates that we are inclined to consume renewable power first. This is because renewable power is free while grid power has cost. In addition, due to the limited energy storage size, we may suffer from renewable energy loss when the energy queue size is large. Therefore, it is preferable to keep the size of the energy queue small.

Based on Lemma 1, without loss of optimality, we can first solve Problem 10ver a reduced policy $\pi=\left\{\Omega^{1}, \Omega^{2}, \cdots\right\}$, where $\Omega^{t}=\left(\Omega_{p}^{t}, \Omega_{s}^{t}\right)$ and then obtain the optimal $\Omega_{p}^{E, t}$ and $\Omega_{p}^{G, t}$ from the optimal $\Omega_{p}^{t}$ using Lemma 1

Next, we exploit the i.i.d. property of the CSI to reduce the state space. We first define partitioned actions below:

Definition 2 (Partitioned Actions): Given $\Omega^{t}=\left(\Omega_{p}^{t}, \Omega_{s}^{t}\right)$, we define

$\Omega^{t}(\mathbf{E}, \mathbf{Q})=\left\{(\mathbf{p}, \mathbf{s})=\left(\Omega_{p}^{t}(\mathbf{E}, \mathbf{Q}), \Omega_{s}^{t}(\mathbf{E}, \mathbf{Q}, \mathbf{H})\right): \mathbf{H} \in \mathcal{H}\right\}, \Omega_{s}^{t}(\mathbf{E}, \mathbf{Q})=\left\{\mathbf{s}=\Omega_{s}^{t}(\mathbf{E}, \mathbf{Q}, \mathbf{H}): \mathbf{H} \in \mathcal{H}\right\}$ as the collection of actions $(\mathbf{p}, \mathbf{s})$ and $\mathbf{s}$ for all possible CSI $\mathbf{H}$ conditioned on a given ESI and QSI pair $(\mathbf{E}, \mathbf{Q}) . \Omega^{t}$ and $\Omega_{s}^{t}$ are therefore equal to the union of all partitioned actions. i.e. $\Omega=\bigcup_{(\mathbf{E}, \mathbf{Q})} \Omega(\mathbf{E}, \mathbf{Q})$ and $\Omega_{s}=\bigcup_{(\mathbf{E}, \mathbf{Q})} \Omega_{s}(\mathbf{E}, \mathbf{Q})$.

Based on Lemma 1 and Definition 2, the optimal control policy in Problem 1 can be obtained by solving an equivalent Bellman equation over a reduced state space, which is summarized in the lemma below.

${ }^{8} \mathrm{POMDP}$ is an extension of MDP when the control agent does not have direct observation of the entire system state. 
Lemma 2 (Equivalent Bellman Equation for POMDP): The optimal control policy for Problem 1 can be obtained by solving the following equivalent Bellman equation w.r.t. $(\theta,\{V(\mathbf{E}, \mathbf{Q})\})$ :

$$
\begin{gathered}
\theta+V(\mathbf{E}, \mathbf{Q})=\min _{\Omega(\mathbf{E}, \mathbf{Q})}\left\{g((\mathbf{E}, \mathbf{Q}), \Omega(\mathbf{E}, \mathbf{Q}))+\sum_{\left(\mathbf{E}^{\prime}, \mathbf{Q}^{\prime}\right)} \operatorname{Pr}\left[\left(\mathbf{E}^{\prime}, \mathbf{Q}^{\prime}\right) \mid(\mathbf{E}, \mathbf{Q}), \Omega(\mathbf{E}, \mathbf{Q})\right] V\left(\mathbf{E}^{\prime}, \mathbf{Q}^{\prime}\right)\right\} \\
\forall(\mathbf{E}, \mathbf{Q}) \in \mathcal{E} \times \mathcal{Q}
\end{gathered}
$$

where $g((\mathbf{E}, \mathbf{Q}), \Omega(\mathbf{E}, \mathbf{Q}))=\sum_{k \in \mathcal{K}} \beta_{k} f\left(Q_{k}\right)+\sum_{b \in \mathcal{B}} \gamma_{b} \Omega_{p}(\mathbf{E}, \mathbf{Q}) \mathbf{I}\left[E_{b}=0\right]$ is the per-stage cost function, $\operatorname{Pr}\left[\left(\mathbf{E}^{\prime}, \mathbf{Q}^{\prime}\right) \mid(\mathbf{E}, \mathbf{Q}), \Omega(\mathbf{E}, \mathbf{Q})\right]=\mathbb{E}\left[\operatorname{Pr}\left[\left(\mathbf{E}^{\prime}, \mathbf{Q}^{\prime}\right) \mid \chi, \Omega(\boldsymbol{\chi})\right] \mid(\mathbf{E}, \mathbf{Q})\right]$ is the transition kernel. $\theta$ is the optimal value for all $\chi$, i.e., $\theta=\min _{\pi} J_{\pi}^{(\boldsymbol{\beta}, \gamma)}(\chi) \forall \chi \in \mathcal{X}$ and $\{V(\mathbf{E}, \mathbf{Q})\}$ is called the potential function. Furthermore, if $\Omega^{*}(\mathbf{E}, \mathbf{Q})=\left(\Omega_{p}^{*}(\mathbf{E}, \mathbf{Q}), \Omega_{s}^{*}(\mathbf{E}, \mathbf{Q})\right)$ attains the minimum of the R.H.S. of $(10)$ for all $(\mathbf{E}, \mathbf{Q}) \in \mathcal{E} \times \mathcal{Q}$, the stationary policy $\Omega^{*}=\left(\Omega_{p}^{*}, \Omega_{s}^{*}\right)$ is optimal (i.e., $\left.\pi^{*}=\left\{\Omega^{*}, \Omega^{*}, \cdots\right\}\right)$.

Proof: Please refer to the Appendix B.

Remark 5 (Interpretation of Equivalent Bellman Equation): The equivalent Bellman equation in (10) is defined on the reduced space of the ESI and QSI (E, Q) only. Nevertheless, by solving (10), we can obtain a stationary BS-DTX policy $\Omega_{p}^{*}$, which is a function of (ESI, QSI), and a stationary user scheduling policy $\Omega_{s}^{*}$, which is a function of (ESI, QSI, CSI).

\section{Centralized Optimal BS-DTX Control and User Scheduling}

To facilitate the BS-DTX control, which is only adaptive to the ESI and the QSI, we introduce the $B S$-DTX control $Q$-factor $\mathbb{Q}(\mathbf{E}, \mathbf{Q}, \mathbf{p})$ w.r.t. the BS-DTX control action p. Based on Lemma 2, we summarize the optimal BS-DTX control in the following corollary.

Corollary 1 (Optimal BS-DTX Control): The optimal BS-DTX control is given by

$$
\Omega_{p}^{*}(\mathbf{E}, \mathbf{Q})=\arg \min _{\mathbf{p} \in \mathcal{P}} \mathbb{Q}(\mathbf{E}, \mathbf{Q}, \mathbf{p}), \forall(\mathbf{E}, \mathbf{Q}) \in \mathcal{E} \times \mathcal{Q}
$$

where $\mathbb{Q}(\mathbf{E}, \mathbf{Q}, \mathbf{p})$ is the BS-DTX control Q-factor given by the following Bellman equation w.r.t. $(\theta,\{\mathbb{Q}(\mathbf{E}, \mathbf{Q}, \mathbf{p})\}):$

$$
\begin{aligned}
& \theta+\mathbb{Q}(\mathbf{E}, \mathbf{Q}, \mathbf{p}) \\
= & \left.\min _{\Omega_{s}(\mathbf{E}, \mathbf{Q})}\left\{g((\mathbf{E}, \mathbf{Q}) \in \mathcal{E} \times \mathbf{Q}), \mathbf{p}, \Omega_{s}(\mathbf{E}, \mathbf{Q})\right)+\sum_{\left(\mathbf{E}^{\prime}, \mathbf{Q}^{\prime}\right)} \operatorname{Pr}\left[\left(\mathbf{E}^{\prime}, \mathbf{Q}^{\prime}\right) \mid(\mathbf{E}, \mathbf{Q}), \mathbf{p}, \Omega_{s}(\mathbf{E}, \mathbf{Q})\right] \min _{\mathbf{p}^{\prime} \in \mathcal{P}} \mathbb{Q}\left(\mathbf{E}^{\prime}, \mathbf{Q}^{\prime}, \mathbf{p}^{\prime}\right)\right\}
\end{aligned}
$$

Proof: Please refer to Appendix B.

As the distributions of the energy and data arrival processes are unknown to the controllers, we introduce the post-decision state potential function $U(\widetilde{\mathbf{E}}, \widetilde{\mathbf{Q}})$ to determine the user selection [17]. The post-decision state $(\widetilde{\mathbf{E}}, \widetilde{\mathbf{Q}})$ is defined to be the virtual partial system state immediately after making 
an action before the new renewable energy and data arrive9. Based on Lemma 2, we summarize the optimal user scheduling in the following corollary.

Corollary 2 (Optimal User Scheduling): The optimal user scheduling is given by

$$
\begin{gathered}
\Omega_{s}^{*}(\boldsymbol{\chi})=\arg \min _{\mathbf{s} \in \mathcal{S}\left(\mathbf{p}^{*}\right)}\left\{\sum_{\mathbf{d} \in \mathcal{D}}\left(\prod_{k \in \mathcal{K}}\left(1-d_{k}-(-1)^{d_{k}} \operatorname{Pr}\left[\rho_{k}\left(\mathbf{H}, \mathbf{p}^{*}, \mathbf{s}\right) \geq \delta_{k}\right]\right) U\left(\left[\mathbf{E}-\mathbf{p}^{*}\right]^{+},[\mathbf{Q}-\mathbf{d}]^{+}\right)\right)\right\} \\
\forall \boldsymbol{\chi} \in \mathcal{X}
\end{gathered}
$$

where $\mathbf{p}^{*}=\Omega_{p}^{*}(\mathbf{E}, \mathbf{Q})$ is the optimal BS-DTX control action given by $111, \mathcal{S}(\mathbf{p}) \triangleq\{\mathbf{s} \in \mathcal{S}$ : $\left.\sum_{k \in \mathcal{K}_{b}} s_{k}=p_{b}, b \in \mathcal{B}\right\}$ denotes the feasible user scheduling action space under the BS-DTX control action $\mathbf{p}, d_{k} \in \mathcal{D} \triangleq\{0,1\}$, and $\mathbf{d}=\left\{d_{k} \in \mathcal{D}_{k}: k \in \mathcal{K}\right\} \in \mathcal{D} \triangleq \mathcal{D}^{K} \cdot U(\widetilde{\mathbf{E}}, \widetilde{\mathbf{Q}})$ is the post-decision potential function given by the following Bellman equation w.r.t. $(\theta,\{U(\widetilde{\mathbf{E}}, \widetilde{\mathbf{Q}})\})$ [17]:

$$
\begin{aligned}
& \theta+U(\widetilde{\mathbf{E}}, \widetilde{\mathbf{Q}}) \\
= & \sum_{\mathbf{A}^{E}, \mathbf{A}^{Q}} \operatorname{Pr}\left[\mathbf{A}^{E}\right] \operatorname{Pr}\left[\mathbf{A}^{Q}\right] \min _{\Omega(\mathbf{E}, \mathbf{Q})}\left\{g((\mathbf{E}, \mathbf{Q}), \Omega(\mathbf{E}, \mathbf{Q}))+\sum_{\left(\widetilde{\mathbf{E}}^{\prime}, \widetilde{\mathbf{Q}}^{\prime}\right)} \operatorname{Pr}\left[\left(\widetilde{\mathbf{E}}^{\prime}, \widetilde{\mathbf{Q}}^{\prime}\right) \mid(\mathbf{E}, \mathbf{Q}), \Omega(\mathbf{E}, \mathbf{Q})\right] U\left(\widetilde{\mathbf{E}}^{\prime}, \widetilde{\mathbf{Q}}^{\prime}\right)\right\}
\end{aligned}
$$

where $\mathbf{E}=\min \left\{\widetilde{\mathbf{E}}+\mathbf{A}^{E}, N_{E}\right\}$ and $\mathbf{Q}=\min \left\{\widetilde{\mathbf{Q}}+\mathbf{A}^{Q}, N_{Q}\right\}$.

Proof: Please refer to Appendix B.

Remark 6 (Complexity of Centralized Delay-Optimal Solution): The complexity of obtaining the original Q-factor and the associated BS-DTX control is $\mathcal{O}\left(\left(N_{E}+1\right)^{B}\left(N_{Q}+1\right)^{K} 2^{B}\right)$. The complexity of obtaining the original post-decision state potential function and the associated user scheduling is $\mathcal{O}\left(\left(N_{E}+1\right)^{B}\left(N_{Q}+1\right)^{K}\right)$.

\section{Low Complexity Delay-AWARe Distributed Solution}

Obtaining the optimal control in (11) and (13) has exponential complexity and requires centralized implementation at the BSC and knowledge of the aggregation of the ESI, QSI and CSI, which leads to huge signaling overhead. In this section, we shall first introduce a randomized base policy. Based on that, we shall propose a low complexity distributed deterministic policy using approximate dynamic programming [11]. We shall show that the proposed solution has better performance than the randomized base policy.

${ }^{9}$ For example, $\chi=(\mathbf{E}, \mathbf{Q}, \mathbf{H})$ is the state at the beginning of some slot (also called the pre-decision state) and making an action $(\mathbf{p}, \mathbf{s})=\Omega(\chi)$ leads to $\boldsymbol{\rho}=\left\{\rho_{k}: k \in \mathcal{K}\right\}$ with $\rho_{k}$ given by 1 . Then, the post-decision state immediately after the action is $\widetilde{\chi}=(\widetilde{\mathbf{E}}, \widetilde{\mathbf{Q}}, \mathbf{H})$, where $\widetilde{\mathbf{E}}=[\mathbf{E}-\mathbf{p}]^{+}$and $\widetilde{\mathbf{Q}}=[\mathbf{Q}-\mathbf{I}[\boldsymbol{\rho} \succeq \boldsymbol{\delta}]]^{+}$, where $\boldsymbol{\delta}=\left\{\delta_{k}: k \in \mathcal{K}\right\}$. If new arrivals $\mathbf{A}^{E}$ and $\mathbf{A}^{Q}$ occur in the post-decision state, and the CSI changes to $\mathbf{H}^{\prime}$, then the system reaches the next actual state, i.e., pre-decision state $\chi^{\prime}=\left(\min \left\{\widetilde{\mathbf{E}}+\mathbf{A}^{E}, N_{E}\right\}, \min \left\{\widetilde{\mathbf{Q}}+\mathbf{A}^{Q}, N_{Q}\right\}, \mathbf{H}^{\prime}\right)$. 


\section{A. Randomized Base Policy}

We first introduce a randomized base policy and discuss an important structural property of the equivalent Bellman equations in (12) and (14)) under this base policy.

Definition 3 (Randomized Base Policy): A randomized base policy is denoted as $\hat{\Omega}=\left(\hat{\Omega}_{p}, \hat{\Omega}_{s}\right)$. The randomized base policy for BS-DTX control $\hat{\Omega}_{p}$ is given by a distribution on the action space of $\mathbf{p}$, i.e., $\mathcal{P}$. The randomized base policy for user scheduling $\hat{\Omega}_{s}$ is given by a mapping from the CSI $\mathbf{H}$ to a probability distribution $\hat{\Omega}_{s}(\mathbf{H})$ on the action space of s, i.e., $\mathcal{S}$.

Under a randomized base policy, the corresponding Q-factor and post-decision potential function have the following decomposition structure.

Lemma 3 (Decomposition under Randomized Base Policy): Given any randomized base policy $\hat{\Omega}$, the Q-factor $\hat{\mathbb{Q}}(\mathbf{E}, \mathbf{Q}, \mathbf{p})$ and the potential function $\hat{U}(\widetilde{\mathbf{E}}, \widetilde{\mathbf{Q}})$ associated with the equivalent Bellman equations in (12) and (14) can be expressed as: $\hat{\mathbb{Q}}(\mathbf{E}, \mathbf{Q}, \mathbf{p})=\sum_{b \in \mathcal{B}} \sum_{k \in \mathcal{K}_{b}} \hat{\mathbb{Q}}_{k}\left(E_{b}, Q_{k}, \mathbf{p}\right)$ and $\hat{U}(\widetilde{\mathbf{E}}, \widetilde{\mathbf{Q}})=\sum_{b \in \mathcal{B}} \sum_{k \in \mathcal{K}_{b}} \hat{U}_{k}\left(\widetilde{E}_{b}, \widetilde{Q}_{k}\right)$, where

$$
\begin{aligned}
& \hat{\theta}_{k}+\hat{\mathbb{Q}}_{k}\left(E_{b}, Q_{k}, \mathbf{p}\right) \\
= & \hat{g}_{k}\left(E_{b}, Q_{k}, p_{b}\right)+\sum_{\left(E_{b}^{\prime}, Q_{k}^{\prime}\right)} \hat{\operatorname{Pr}}\left[\left(E_{b}^{\prime}, Q_{k}^{\prime}\right) \mid\left(E_{b}, Q_{k}\right), \mathbf{p}\right] \mathbb{E}^{\hat{\Omega}_{p}}\left[\mathbb{Q}_{k}\left(E_{b}^{\prime}, Q_{k}^{\prime}, \mathbf{p}^{\prime}\right)\right] \\
& \hat{\theta}_{k}+\hat{U}_{k}\left(\widetilde{E}_{b}, \widetilde{Q}_{k}\right) \\
= & \sum_{\left(A_{b}^{E}, A_{k}^{Q}\right)} \operatorname{Pr}\left[\widetilde{E}_{b}^{E} \in \mathcal{E}, \widetilde{Q}_{k} \in \mathcal{Q} \operatorname{Pr}\left[A_{k}^{Q}\right]\left(\mathbb{E}^{\hat{\Omega}_{p}}\left[\hat{g}_{k}\left(E_{b}, Q_{k}, p_{b}\right)\right]+\sum_{\left(\widetilde{E}_{b}^{\prime}, \widetilde{Q}_{k}^{\prime}\right)} \mathbb{E}^{\hat{\Omega}_{p}}\left[\operatorname{Pr}\left[\left(\widetilde{E}_{b}^{\prime}, \widetilde{Q}_{k}^{\prime}\right) \mid\left(E_{b}, Q_{k}\right), \mathbf{p}\right]\right] \widetilde{V}_{k}\left(\widetilde{\mathbf{E}}_{n}^{\prime}, \widetilde{Q}_{k}^{\prime}\right)\right)\right.
\end{aligned}
$$

with $\hat{g}_{k}\left(E_{b}, Q_{k}, p_{b}\right)=\beta_{k} f\left(Q_{k}\right)+\gamma_{b} p_{b} \mathbf{I}\left[E_{b}=0\right] \mathbb{E}\left[\hat{\operatorname{Pr}}\left[s_{k}=1 \mid \mathbf{H}\right]\right]$ and $\hat{\operatorname{Pr}}\left[\left(E_{b}^{\prime}, Q_{k}^{\prime}\right) \mid\left(E_{b}, Q_{k}\right), \mathbf{p}\right]=$ $\mathbb{E}\left[\mathbb{E}^{\hat{\Omega}_{s}}\left[\operatorname{Pr}\left[\left(E_{b}^{\prime}, Q_{k}^{\prime}\right) \mid\left(E_{b}, Q_{k}, \mathbf{H}\right), p_{b}, s_{k}\right] \mid \mathbf{H}\right]\right]$.

Proof: Please refer to Appendix C.

\section{B. Low Complexity Delay-aware Distributed Solution}

Based on the randomized base policy $\hat{\Omega}$, we shall obtain a low complexity distributed deterministic policy $\hat{\Omega}^{*}$ by Q-factor and potential function approximation. The solution is elaborated below.

1) BS-DTX Control Policy Over a Longer Timescale: To reduce the complexity and to facilitate distributed implementation, we approximate the BS-DTX control Q-factor $\mathbb{Q}(\mathbf{E}, \mathbf{Q}, \mathbf{p})$ in (12) by $\hat{\mathbb{Q}}(\mathbf{E}, \mathbf{Q}, \mathbf{p})$, i.e.,

$$
\mathbb{Q}(\mathbf{E}, \mathbf{Q}, \mathbf{p}) \approx \hat{\mathbb{Q}}(\mathbf{E}, \mathbf{Q}, \mathbf{p})=\sum_{b \in \mathcal{B}} \sum_{k \in \mathcal{K}_{b}} \hat{\mathbb{Q}}_{k}\left(E_{b}, Q_{k}, \mathbf{p}\right)
$$


where $\hat{\mathbb{Q}}_{k}\left(E_{b}, Q_{k}\right)$ is given by the per-flow fixed point equation in (15). The BSC determines the BS-DTX control based on the aggregation of the ESI and QSI according to

$$
\hat{\mathbf{p}}^{*}(\mathbf{E}, \mathbf{Q})=\arg \min _{\mathbf{p} \in \mathcal{P}} \sum_{b \in \mathcal{B}} \sum_{k \in \mathcal{K}_{b}} \hat{\mathbb{Q}}_{k}\left(E_{b}, Q_{k}, \mathbf{p}\right)
$$

Remark 7 (Complexity of the BS-DTX Control): Under the linear Q-factor approximation in (17), the complexity of obtaining the BS-DTX control is reduced from $\mathcal{O}\left(\left(N_{E}+1\right)^{B}\left(N_{Q}+1\right)^{K} 2^{B}\right)$ to $\mathcal{O}\left(\left(N_{E}+1\right)\left(N_{Q}+1\right) 2^{B} K\right)$. To further reduce the complexity w.r.t. $B$, we can partition the BSs into macro-groups with size $N_{B}$. The BS-DTX control in (18) can be done for each of the $\frac{B}{N_{B}}$ macrogroups separately [16]. In practice, $N_{B} \ll B$ and hence, the complexity becomes $\mathcal{O}\left(\left(N_{E}+1\right)\left(N_{Q}+\right.\right.$ 1) $2^{N_{B}} \frac{B}{N_{B}} K$ ), which is linear w.r.t. $B$.

2) Distributed User Scheduling Policy at the CM Over a Shorter Timescale: To reduce the complexity and to facilitate distributed implementation of the user scheduling, we approximate the postdecision state potential function $U(\widetilde{\mathbf{E}}, \widetilde{\mathbf{Q}})$ in $(14)$ by $\hat{U}(\widetilde{\mathbf{E}}, \widetilde{\mathbf{Q}})$, i.e.,

$$
U(\widetilde{\mathbf{E}}, \widetilde{\mathbf{Q}}) \approx \hat{U}(\widetilde{\mathbf{E}}, \widetilde{\mathbf{Q}})=\sum_{b \in \mathcal{B}} \sum_{k \in \mathcal{K}_{b}} \hat{U}_{k}\left(E_{b}, Q_{k}\right)
$$

where $\hat{U}_{k}\left(\widetilde{E}_{b}, \widetilde{Q}_{k}\right)$ is given by the per-flow fixed point equation in (16). Substituting the approximation in (19) into the optimal user scheduling in (13), the user scheduling solution under the approximation is summarized below.

Lemma 4 (Distributed User Scheduling): Under the linear potential function approximation in (19), the distributed user scheduling action $\hat{\mathbf{s}}_{n}^{*}$ of the $n$-th cluster based on the per-cluster ESI, QSI and CSI under $\hat{\mathbf{p}}^{*}(\mathbf{E}, \mathbf{Q})$ obtained by 18 is given by

$$
\begin{aligned}
& \hat{\mathbf{s}}_{n}^{*}\left(\mathbf{E}_{n}, \mathbf{Q}_{n}, \mathbf{H}_{n}\right), \\
= & \arg \max _{\mathbf{s}_{n} \in \mathcal{S}_{n}\left(\hat{\mathbf{p}}_{n}^{*}\right)} \sum_{k \in \mathcal{K}_{n}} s_{k} \operatorname{Pr}\left[\rho_{k}\left(\mathbf{H}_{n}, \hat{\mathbf{p}}_{n}^{*}, \mathbf{s}_{n}\right) \geq \delta_{k}\right]\left(\hat{U}_{k}\left(\left[E_{b}-\hat{p}_{b}^{*}\right]^{+}, Q_{k}\right)-\hat{U}_{k}\left(\left[E_{b}-\hat{\mathcal{H}}_{b}^{*}\right]^{+},\left[Q_{k}-1\right]^{+}\right)\right)
\end{aligned}
$$

where $\mathcal{S}_{n}\left(\mathbf{p}_{n}\right) \triangleq\left\{\mathbf{s}_{n} \in \mathcal{S}^{\sum_{b \in \mathcal{B}_{n}} K_{b}}: \sum_{k \in \mathcal{K}_{b}} s_{k}=p_{b}, b \in \mathcal{B}_{n}\right\}$ denotes the feasible user scheduling action space of cluster $n$ under the BS-DTX control action $\mathbf{p}_{n}$.

Proof: Please refer to Appendix D.

Remark 8 (Complexity of the User Scheduling): The user scheduling action in (20) is a function of the per-cluster ESI, QSI and CSI, and is computed locally at the $n$-th CM. Under the linear potential function approximation in 199), the complexity of user scheduling is reduced from $\mathcal{O}\left(\left(N_{E}+1\right)^{B}\left(N_{Q}+\right.\right.$ $\left.1)^{K}\right)$ to $\mathcal{O}\left(\left(N_{E}+1\right)\left(N_{Q}+1\right) K\right)$.

\section{Performance of Low Complexity Delay-aware Distributed Solution}

The key motivation of the linear approximatios of the Q-function and potential function in (17) and (19) is to facilitate distributed control. The following theorem shows that the proposed distributed policy always achieves better performance than the randomized base policy. 
Theorem 1 (Performance Improvement): If $\operatorname{Pr}\left[\left(\mathbf{E}^{\prime}, \mathbf{Q}^{\prime}\right) \mid(\mathbf{E}, \mathbf{Q}),(\mathbf{p}, \mathbf{s})\right] \neq \operatorname{Pr}\left[\left(\mathbf{E}^{\prime}, \mathbf{Q}^{\prime}\right) \mid(\mathbf{E}, \mathbf{Q}),\left(\mathbf{p}^{\prime}, \mathbf{s}^{\prime}\right)\right]$ for any $(\mathbf{p}, \mathbf{s}) \neq\left(\mathbf{p}^{\prime}, \mathbf{s}^{\prime}\right)$ and $(\mathbf{E}, \mathbf{Q}) \in \mathcal{E} \times \mathcal{Q}$, then we have $\hat{\theta}^{*}(\mathbf{E}, \mathbf{Q})<\hat{\theta}$ for all $(\mathbf{E}, \mathbf{Q}) \in \mathcal{E} \times \mathcal{Q}$, where $\hat{\theta}^{*}(\mathbf{E}, \mathbf{Q})$ is the average cost under the proposed solution starting from state $(\mathbf{E}, \mathbf{Q})$ and $\hat{\theta}$ is the average cost under any randomized base policy, respectively.

Proof: Please refer to Appendix E.

\section{Distributed Online Learning Via Stochastic Approximation}

Observe that the BS-DTX control and the user scheduling in (18) and (20) require the knowledge of $\left\{\hat{\mathbb{Q}}_{k}\left(E_{b}, Q_{k}, \mathbf{p}\right)\right\}$ and $\left\{\hat{U}_{k}\left(\widetilde{E}_{b}, \widetilde{Q}_{k}\right)\right\}$, respectively, which are defined in the fixed point equations in (15) and (16), respectively. However, solving these fixed point equations is also quite challenging. In this section, we shall propose an online distributed stochastic learning [18] algorithm to estimate $\left\{\hat{\mathbb{Q}}_{k}\left(E_{b}, Q_{k}, \mathbf{p}\right)\right\}$ and $\left\{\hat{U}_{k}\left(\widetilde{E}_{b}, \widetilde{Q}_{k}\right)\right\}$ using the per-cluster system state information only. We shall prove that the proposed distributed algorithm converges almost surely to the fixed point solutions.

\section{A. Distributed Online Learning for $\left\{\hat{\mathbb{Q}}_{k}\left(E_{b}, Q_{k}, \mathbf{p}\right)\right\}$ and $\left\{\hat{U}_{k}\left(\widetilde{E}_{b}, \widetilde{Q}_{k}\right)\right\}$}

Since the statistics of $\mathbf{A}^{Q}(t)$ and $\mathbf{A}^{E}(t)$ are unknown to the controller, instead of computing $\left\{\hat{\mathbb{Q}}_{k}\left(E_{b}, Q_{k}, \mathbf{p}\right)\right\}$ and $\left\{\hat{U}_{k}\left(\widetilde{E}_{b}, \widetilde{Q}_{k}\right)\right\}$ of a chosen $\hat{\Omega}$ offline, we shall estimate them distributively at each BS based on the instantaneous observations.

Algorithm 1: (Online Per-User Q-factor and Potential Function Learning Algorithm)

- Step 1 [Initialization at the BSs]: Set $t=0$. Each BS $b$ initializes $\left\{\hat{\mathbb{Q}}_{k}^{0}\left(E_{b}, Q_{k}, \mathbf{p}\right)\right\}$ and $\left\{\hat{U}_{k}^{0}\left(\widetilde{E}_{b}, \widetilde{Q}_{k}\right)\right\}$ for all $k \in \mathcal{K}_{b}$.

- Step 2 [BS-DTX Control at the BSC]: At the beginning of the $t$-th slot, each BS $b$ reports $\left\{\sum_{k \in \mathcal{K}_{b}} \hat{\mathbb{Q}}_{k}^{t}\left(E_{b}(t), Q_{k}(t), \mathbf{p}\right): \mathbf{p} \in \mathcal{P}\right\}$ to the BSC. The BSC determines BS-DTX control $\hat{\mathbf{p}}^{*}(t) \triangleq \hat{\mathbf{p}}^{*}(\mathbf{E}(t), \mathbf{Q}(t))$ according to (18) and broadcasts $\hat{\mathbf{p}}^{*}(t)$ to all the CMs. Each CM $n$ informs $\hat{p}_{b}^{*}(t)$ to each BS $b \in \mathcal{B}_{n}$. Each BS $b$ determines its renewable and grid power allocations, i.e., $\hat{p}_{b}^{E *}(t)=\hat{p}_{b}^{*}(t) \mathbf{I}\left[E_{b}(t)>0\right]$ and $\hat{p}_{b}^{G *}(t)=\hat{p}_{b}^{*}(t) \mathbf{I}\left[E_{b}(t)=0\right]$, respectively.

- Step 3 [User Scheduling at the CMs]: Each BS $b$ reports $\left\{\hat{U}_{k}^{t}\left(E_{b}(t), Q_{k}(t)\right): k \in \mathcal{K}_{b}\right\}$ to its CM. Each CM $n$ determines user selection $\hat{\mathbf{s}}_{n}^{*}(t) \triangleq \hat{\mathbf{s}}_{n}^{*}\left(\mathbf{E}_{n}(t), \mathbf{Q}_{n}(t), \mathbf{H}_{n}(t)\right)$ according to (20) under given BS-DTX control $\hat{\mathbf{p}}^{*}(t)$.

- Step 4 [Per-flow Q-factor and Potential Function Update at the BSs]: Based on the current observations $A_{b}^{E}(t)$ and $A_{k}^{Q}(t)\left(k \in \mathcal{K}_{b}\right)$, each BS $b$ updates the per-flow Q-factor and potential 
function for the MSs in its cell according to (21) and (22) for all $k \in \mathcal{K}_{b}$.

$$
\begin{aligned}
& \hat{\mathbb{Q}}_{k}^{t+1}\left(E_{b}, Q_{k}, \mathbf{p}\right) \\
= & \hat{\mathbb{Q}}_{k}^{t}\left(E_{b}, Q_{k}, \mathbf{p}\right)+\epsilon_{t}\left[F_{k}\left(\hat{\mathbb{Q}}_{k}^{t}, E_{b}, Q_{k}, \mathbf{p}\right)-F_{k}\left(\hat{\mathbb{Q}}_{k}^{t}, E_{b}^{I}, Q_{k}^{I}, \mathbf{p}^{I}\right)-\mathbb{Q}_{k}^{t}\left(E_{b}, Q_{k}, \mathbf{p}\right)\right] \\
& \hat{U}_{k}^{t+1}\left(\widetilde{E}_{b}, \widetilde{Q}_{k}\right) \\
= & \hat{U}_{k}^{t}\left(\widetilde{E}_{b}, \widetilde{Q}_{k}\right)+\epsilon_{t}\left[T_{k}\left(\hat{\mathbf{U}}_{k}^{t}, \widetilde{E}_{b}, \widetilde{Q}_{k}\right)-\widetilde{Q}_{k} \in \mathcal{Q}\left(\hat{\mathbf{U}}_{k}^{t}, \widetilde{E}_{b}^{I}, \widetilde{Q}_{k}^{I}\right)-\hat{U}_{k}^{t}\left(\widetilde{E}_{b}, \widetilde{Q}_{k}\right)\right]
\end{aligned}
$$

where

$$
\begin{gathered}
F_{k}\left(\hat{\mathbb{Q}}_{k}^{t}, E_{b}, Q_{k}, \mathbf{p}\right)=\hat{g}_{k}\left(E_{b}, Q_{k}, p_{b}\right)+\sum_{\left(E_{b}^{\prime}, Q_{k}^{\prime}\right)} \hat{\operatorname{Pr}}\left[\left(E_{b}^{\prime}, Q_{k}^{\prime}\right) \mid\left(E_{b}, Q_{k}\right), \mathbf{p}\right] \\
\times \mathbb{E}^{\hat{\Omega}_{p}}\left[\mathbb{Q}_{k}^{t}\left(\min \left\{E_{b}^{\prime}+A_{b}^{E}(t), N_{E}\right\}, \min \left\{Q_{k}^{\prime}+A_{k}^{Q}(t), N_{Q}\right\}, \mathbf{p}^{\prime}\right)\right] \\
T_{k}^{t}\left(\hat{\mathbf{U}}_{k}^{t}, \widetilde{E}_{b}, \widetilde{Q}_{k}\right)=\mathbb{E}^{\hat{\Omega}_{p}}\left[\hat{g}_{k}\left(\min \left\{\widetilde{E}_{b}+A_{b}^{E}(t), N_{E}\right\}, \min \left\{\widetilde{Q}_{k}+A_{k}^{Q}(t), N_{Q}\right\}, p_{b}\right)\right] \\
+\sum_{\left(\widetilde{E}_{b}^{\prime}, \widetilde{Q}_{k}^{\prime}\right)} \mathbb{E}^{\hat{\Omega}_{p}}\left[\hat{\operatorname{Pr}}\left[\left(\widetilde{E}_{b}^{\prime}, \widetilde{Q}_{k}^{\prime}\right) \mid\left(\min \left\{\widetilde{E}_{b}+A_{b}^{E}(t), N_{E}\right\}, \min \left\{\widetilde{Q}_{k}+A_{k}^{Q}(t), N_{Q}\right\}\right), \mathbf{p}\right]\right] \hat{U}_{k}^{t}\left(\widetilde{E}_{b}^{\prime}, \widetilde{Q}_{k}^{\prime}\right)
\end{gathered}
$$

$E_{b}^{\prime}=\left[E_{b}-p_{b}\right]^{+}, Q_{k}^{\prime}=\left[Q_{k}-\mathbf{I}\left[\rho_{k}\left(\mathbf{H}_{n}, \mathbf{p}, \mathbf{s}_{n}\right) \geq \delta_{k}\right]\right]^{+}, \widetilde{E}_{b}^{\prime}=\left[\min \left\{\widetilde{E}_{b}+A_{b}^{E}(t), N_{E}\right\}-p_{b}\right]^{+}$, $\widetilde{Q}_{k}^{\prime}=\left[\min \left\{\widetilde{Q}_{k}+A_{k}^{Q}(t), N_{Q}\right\}-\mathbf{I}\left[\rho_{k}\left(\mathbf{H}_{n}, \mathbf{p}, \mathbf{s}_{n}\right) \geq \delta_{k}\right]\right]^{+} \cdot \mathbf{p}^{I}$ is the reference BS-DTX control action and $E_{b}^{I}, Q_{k}^{I}, \widetilde{E}_{b}^{I}, \widetilde{Q}_{k}^{I}$ are the reference state 10 for the Q-factor update in (21) and the potential function update in (22), respectively. $\left\{\epsilon_{t}\right\}$ are diminishing positive step size sequences satisfying the following conditions: $\epsilon_{t} \geq 0, \sum_{t} \epsilon_{t}=\infty, \sum_{t} \epsilon_{t}^{2}<\infty$.

\section{B. Performance of the Distributed Learning Algorithm}

The convergence of Algorithm 1 is summarized below.

Lemma 5 (Convergence of Algorithm 廿): The iterative updates of the per-flow Q-factor and the per-flow potential function in (21) and (22) converge almost surely, i.e., $\lim _{t \rightarrow \infty} \mathbb{Q}_{k}^{t}=\hat{\mathbb{Q}}_{k}^{\infty}$ a.s. and $\lim _{t \rightarrow \infty} \hat{\mathbf{U}}_{k}^{t}=\hat{\mathbf{U}}_{k}^{\infty}$ a.s. $(\forall k \in \mathcal{K})$, where $\hat{\mathbb{Q}}_{k}^{\infty}$ and $\hat{\mathbf{U}}_{k}^{\infty}$ are the solutions of the fixed point equations in (15) and (16), respectively.

Proof: Please refer to Appendix F.

Remark 9 (Signaling Requirement of Distributed Two-Timescale Algorithm 17):

- Signaling requirement over a short timescale (per slot): Each BS needs to collect the local CSI over the radio interface. The BSs within a cluster also need to report the local CSI to its CM.

\footnotetext{
${ }^{10}$ The reference action and states are used to bootstrap the online learning algorithms [19] for 21] and 22] respectively. Without loss of generality, we set $E_{b}^{I}=0, Q_{k}^{I}=0, \mathbf{p}^{I}=\left\{p_{b}^{I}=1: b \in \mathcal{B}\right\}, \widetilde{E}_{b}^{I}=0$ and $\widetilde{Q}_{k}^{I}=0$.
} 
Yet, the signaling loading and the latency requirement for this part is in fact similar to the existing HSDPA and LTE systems.

- Signaling requirement through the backhaul over a long timescale (in convergent stage):

Each BS needs to report the Q-factors of the (updated) local QSI to the BSC (for the BS-DTX control) as well as the potential functions of the (updated) local QSI to the CM (for the user scheduling within a cluster). These signaling exchanges are over the high-speed backhaul and over a longer timescale (not on a slot by slot basis). The latency of signaling over backhaul (typically less than 10ms) is negligible.

\section{Stability AnAlysis}

In this section, we shall analyze the stability conditions for the data queues in the coordinated MIMO networks with infinite data buffer size $\left(N_{Q}=\infty\right)$ and finite energy storage size $\left(N_{E}<\infty\right)$, and discuss various design insights. We have the following assumption on the BS and MS distributions.

Assumption 5 (BS and MS Distributions): The location of the BSs follows a homogeneous Poisson Point Process (PPP) $\Phi$ of density $\lambda$ and the location of the MSs follows some independent stationary point process in the Euclidean plane [12], [13]. Each MS is associated with the closest BS, i.e., the MSs in the Voronoi cell of a BS are associated with it.

To simplify the analysis, we consider a homogeneous network with $K_{b}=1, P_{b}=P \forall b \in \mathcal{B}$ and $\delta_{k}=\delta \forall k \in \mathcal{K}$. In addition, we assume the CSI follows complex Gaussian fading and the longterm path gain follows standard power law $L_{k, b}=r_{k, b}^{-\alpha}$, where $r_{k, b}$ is the distance between BS $b$ and MS $k$ and $\alpha>2$ is the path loss exponent. Furthermore, the renewable energy and bursty data arrivals under Assumptions 3 and 4 are specialized to Bernoulli processes, i.e., $A_{k}^{Q}(t), A_{b}^{E}(t) \in\{0,1\}$, $\mathbb{E}\left[A_{k}^{Q}(t)\right]=\lambda^{Q}<1$ and $\mathbb{E}\left[A_{b}^{E}(t)\right]=\lambda^{E}<1$ for all $k \in \mathcal{K}$ and $b \in \mathcal{B}$. We consider the following randomized BS-DTX policy.

Definition 4 (Randomized BS-DTX control Policy): At each slot $t$, each BS $b \in \mathcal{B}$ is active with probability $p_{t x}>0$, i.e., $\operatorname{Pr}\left[p_{b}(t)=1\right]=p_{t x}$, if $\sum_{k \in \mathcal{K}_{b}} Q_{k}(t)>0 ; p_{b}(t)=0$ otherwise.

In the following, we shall analyze the sufficient conditions for the queue stability (i.e., $Q_{k}(t)$ having a steady state limiting distribution for $t \rightarrow \infty$ [20]) under the randomized policy in Definition 4 of a randomly chosen user.

\section{A. Stability Analysis for Systems without BS Coordination $\left(N_{t}=1\right)$}

In this case, we consider no cooperation among BSs $\left(N_{t}=1\right)$. Using stochastic geometry [13] and the technique of parallel dominant queues [21], [22], the following lemma summarizes the sufficient 
condition for the queue stability of a randomly chosen MS at a distance $r_{1}$ from its BS 11 .

Lemma 6 (Sufficient Condition for Queue Stability without BS Coordination): The data queue of a randomly chosen MS is stable if

$$
\lambda^{Q}<p_{t x} \exp \left(-C_{1} p_{t x} \lambda-\frac{N_{0}}{P} \delta r_{1}^{\alpha}\right) \triangleq \lambda_{\max }^{Q}\left(p_{t x}, N_{t}\right)
$$

In addition, $\lambda_{\max }^{Q}\left(p_{t x}, N_{t}\right)$ corresponds to the maximum average grid power cost per $\operatorname{BS} \overline{p_{\max }^{G}}\left(p_{t x}, N_{E}\right)=$ $\left(1-f\left(p_{t x}, N_{E}\right)\right) p_{t x} . N_{t}=1, C_{1}=\frac{2}{\alpha-2} \pi r_{1}^{2} \delta$ and

$$
f\left(p_{t x}, N_{E}\right)= \begin{cases}\frac{\left(\lambda^{E} / p_{t x}\right)\left(1-\left(\lambda^{E} / p_{t x}\right)^{N_{E}}\right)}{1-\left(\lambda^{E} / p_{t x}\right)^{N_{E}+1}}, & \lambda^{E} \neq p_{t x} \\ \frac{N_{E}}{N_{E}+1}, & \lambda^{E}=p_{t x}\end{cases}
$$

Proof: Please refer to Appendix G.

Remark 10 (Interpretation of Lemma 6): $f\left(p_{t x}, N_{E}\right)$ can be interpreted as the probability that a energy queue is non-empty in a parallel dominant network 12, i.e., $f\left(p_{t x}, N_{E}\right)=\operatorname{Pr}\left[E_{b}>0\right]$. It can be easily verified from (26) that $f\left(p_{t x}, N_{E}\right)$ increases as $N_{E}$ increases and $\lim _{N_{E} \rightarrow \infty} f\left(p_{t x}, N_{E}\right)=$ $\min \left\{\frac{\lambda^{E}}{p_{t x}}, 1\right\} \triangleq f\left(p_{t x}, \infty\right)$, which corresponds to the case with infinite energy storage size. In addition, $\overline{p_{\max }^{G}}\left(p_{t x}, N_{E}\right)=\operatorname{Pr}\left[p_{b}^{G}=1\right]$.

\section{B. Stability Analysis for Systems with BS Coordination $\left(N_{t}>1\right)$}

In this part, we extend the analysis to the case with BS coordination $\left(N_{t}>1\right)$. For a randomly chosen MS, the interference comes from the active BSs outside its cluster. Hence, we need to consider the distribution of the coordination clusters and the associated analysis is more challenging compared with the case without BS coordination $\left(N_{t}=1\right)$ [12].

Lemma 7 (Sufficient Condition for Queue Stability with BS Coordination): For $N_{t}>\frac{\alpha}{2} 13$ the data queue of a randomly chosen MS in the coordinated MIMO network can be stabilized if

$$
\lambda^{Q}<p_{t x} \exp \left(-C_{N_{t}} p_{t x} \lambda-\frac{N_{0}}{P} \delta r_{1}^{\alpha}\right) \triangleq \lambda_{\max }^{Q}\left(p_{t x}, N_{t}\right)
$$

In addition, $\lambda_{\max }^{Q}\left(p_{t x}, N_{t}\right)$ corresponds to the maximum average grid power cost per $\mathrm{BS} \overline{p_{\max }^{G}}\left(p_{t x}, N_{E}\right)=$ $\left(1-f\left(p_{t x}, N_{E}\right)\right) p_{t x} . C_{N_{t}}=\frac{2}{\alpha-2} \pi r_{1}^{2} \delta \min \left\{1, r_{1}^{\alpha-2} \frac{(\lambda \pi)^{-1+\frac{\alpha}{2}} \Gamma\left(N_{t}-\frac{\alpha}{2}\right)}{\Gamma\left(N_{t}-1\right)}\right\}=\mathcal{O}\left(N_{t}^{1-\frac{\alpha}{2}}\right)$ as $N_{t} \rightarrow \infty$.

Proof: Please refer to Appendix H.

\footnotetext{
${ }^{11}$ When $N_{E} \rightarrow \infty$ and $p_{t x}=1$, the result in 25) reduces to the coverage probability for cellular networks without BS coordination obtained in [12].

${ }^{12}$ In the parallel dominant network, dummy packets are transmitted if a data queue is empty. Thus, the BS-sDTX controls are decoupled from the data queues, i.e., independent of the QSI, and hence, the renewable and grid power consumptions are symmetric across all the BSs.

${ }^{13}$ Note that $N_{t}>\frac{\alpha}{2}$ implies $N_{t}>1$ for most of the cases we are interested in, as wu usually have $2<\alpha<4$ in practical systems.
} 


\section{Optimization of Randomized Policy}

We are interested in maximizing $\lambda_{\max }^{Q}\left(p_{t x}, N_{t}\right)$ under grid power constraint $P^{G}$ w.r.t. the parameter $p_{t x}$ in the randomized control policy for any given $N_{E}>0$ and $N_{t} \geq 1$. Specifically, we have

$$
\begin{aligned}
p_{t x}^{*}\left(N_{E}, N_{t}\right)=\arg \max _{p_{t x} \in[0,1]} \lambda_{\max }^{Q}\left(p_{t x}, N_{t}\right) \\
\text { s.t. } \overline{p_{\max }^{G}}\left(p_{t x}, N_{E}\right) \leq P^{G}
\end{aligned}
$$

Let $\lambda_{\max }^{Q *}\left(N_{E}, N_{t}\right)=\lambda_{\max }^{Q}\left(p_{t x}^{*}, N_{t}\right)$ denote the optimal value of the optimization problem in (28). Let $x^{*}\left(N_{E}\right)$ denote the solution to $\overline{p_{\max }^{G}}\left(x, N_{E}\right)=P^{G}$ for any given $N_{E}>0$. The following theorem summarizes the optimal solution.

Theorem 2 (Optimization Solution for Queue Stability): $p_{t x}^{*}\left(N_{E}, N_{t}\right)=\min \left\{x^{*}\left(N_{E}\right), 1, \frac{1}{C_{N_{t}} \lambda}\right\}$. For any given $N_{t} \geq 1, \lambda_{\max }^{Q *}\left(N_{E}, N_{t}\right)$ is strictly increasing in $N_{E}$ if $x^{*}\left(N_{E}\right)<\min \left\{1, \frac{1}{C_{N_{t}} \lambda}\right\}$ and is a constant for all $N_{E}$ if $x^{*}\left(N_{E}\right) \geq \min \left\{1, \frac{1}{C_{N_{t}} \lambda}\right\}$. For any given $N_{E}>0, \lambda_{\max }^{Q *}\left(N_{E}, N_{t}\right)$ is strictly increasing in $N_{t}$.

Proof: Please refer to Appendix I.

\section{RESUlTS AND DisCUSSIONS}

In this section, we shall discuss the design insights from the analytical results in Section VI] We also compare the delay performance gain of the proposed delay-aware low complexity distributed scheme in Section IV] and Section $\mathrm{V}$ with the following two baseline schemes using simulation.

- Baseline 1 [CSI-based Single Cell Scheme]: Baseline 1 refers to the randomized BS-DTX control and CSI-based user scheduling without BS coordination. Each multi-antenna BS uses maximal ratio combining (MRC) and selects one MS with the maximum successful packet transmission probability based on the observed local CSI.

- Baseline 2 [CSI-based Clustered Coordinated MIMO Scheme]: Baseline 2 refers to the randomized BS-DTX control and CSI-based clustered coordinated MIMO with the same coordinated beamforming as the proposed scheme. Each $\mathrm{CM}$ determines the user scheduling to maximize the sum successful packet transmission probability of each cluster based on the observed per-cluster CSI.

In the simulation, we consider a cellular system with 19 BSs, each has a coverage of $500 \mathrm{~m}$ and 2 mobiles per cell, which distribute uniformly in the cell-edge with range $[400 \mathrm{~m}, 500 \mathrm{~m}]$ from the BS. We apply the Urban Macrocell Model in 3GPP [23] with path loss model given by $P L=$ $34.5+35 \log _{10}(r)$, where $r$ (in $\mathrm{m}$ ) is the distance from the transmitter to the receiver. Each element of $\mathbf{h}_{k, b}$ is $\mathcal{C N}(0,1)$. The total bandwidth is $1 \mathrm{MHz}$. The BS transmit power is $P_{b}=35 \mathrm{dBm}$ for all $b \in \mathcal{B}$, the threshold is $\delta_{k}=0.5$, and $\beta_{k}=1$ for all $k \in \mathcal{K}$. $\gamma_{b}$ is the same for all $b \in \mathcal{B}$. We consider Bernoulli arrival processes for the renewable energy and busty data arrivals. The maximum buffer size $N_{Q}=15$ pcks. 


\section{A. Effect of BS Coordination}

From Theorem 2 , we can see that for any given $N_{E}>0$, the loading supported by the energy harvesting system $\lambda_{\max }^{Q *}\left(N_{E}, N_{t}\right)$ increases as $N_{t}$ increases. Intuitively, the gain comes from BS coordination. Fig. [ illustrates the average delay versus the average transmit power cost for different number of transmit antennas $N_{t}$. It can be observed that the average delay of Baseline 2 and the proposed scheme decreases as $N_{t}$ increases. This demonstrates that BS coordination improves the delay performance.

\section{B. Effect of Energy Buffer Size}

From Theorem 2, we can see that for any given $N_{t} \geq 1$, the loading supported by the energy harvesting system $\lambda_{\max }^{Q *}\left(N_{E}, N_{t}\right)$ increases in $N_{E}$. Specifically, when $x^{*}\left(N_{E}\right)<\min \left\{1, \frac{1}{C_{N_{t}} \lambda}\right\}$, $\lambda_{\max }^{Q *}\left(N_{E}, N_{t}\right)$ increases as $N_{E}$ increases. The intuition is that the above condition corresponds to the power-limited region. By increasing $N_{E}$, more renewable energy can be accumulated due to less renewable energy loss when the energy storage is full, and hence more traffic loading can be supported. However, when $x^{*}\left(N_{E}\right) \geq \min \left\{1, \frac{1}{C_{N_{t}} \lambda}\right\}, \lambda_{\max }^{Q *}\left(N_{E}, N_{t}\right)$ is constant for all $N_{E}>0$. The intuition is that the above condition corresponds to the interference-limited region, in which the traffic loading supported cannot be increased by accumulating more renewable energy through increasing $N_{E}$. Fig. 3 illustrates the average delay versus the energy storage size $N_{E}$ at average transmit grid power 15 $\mathrm{dBm}$. It can be observed that the average delay decreases as the energy storage size increases for all the schemes.

\section{Performance of the Proposed Scheme}

Fig. 4 illustrates the average delay versus per-flow loading (average arrival rate $\lambda_{k}$ ). The average delay of all the schemes increases as the loading increases. The proposed scheme also achieves significant gain over the baselines across a wide range of input loading. Fig. 5 illustrates the convergence property of the proposed distributed online learning algorithm for estimating the per-flow potential function and the per-flow Q-factor. It can be observed that the proposed distributed learning algorithm converges quite fast. Furthermore, the average delay at the the 500-th scheduling slot is 4.1853 pcks, which is much smaller than the other baselines.

\section{SUMMARY}

In this paper, we propose a two-timescale delay-optimal BS-DTX control and user scheduling for energy harvesting downlink coordinated MIMO networks. We show that the two-timescale delayoptimal control problem can be modeled as a POMDP and derive the optimal centralized control. To reduce the complexity and facilitate the distributed implementation, we obtain a distributed solution 
with the BS-DTX control at the BSC based on the aggregation of the ESI and QSI and the user scheduling at each CM based on the per-cluster ESI, QSI and CSI with guaranteed delay performance. We prove the almost-sure convergence of the proposed distributed two-timescale algorithm. Furthermore, we analyze the stability conditions for the data queues in coordinated MIMO networks and discuss various design insights.

\section{APPENDIX}

\section{ApPendix A: Proof of Lemma 1}

We shall prove Lemma 1 using sample path arguments. Let $\left\{\mathbf{A}^{E}(\omega, t)\right\},\left\{\mathbf{A}^{Q}(\omega, t)\right\}$ and $\{\mathbf{H}(\omega, t)\}$ be a given sample path (i.e., $\omega$ ) of energy arrivals, packet arrivals and CSI states. Let $\{\mathbf{p}(t)\}$ and $\{\mathbf{s}(t)\}$ be any given sequences of feasible BS-DTX control actions and user scheduling actions. Note that for given $\{\mathbf{p}(t)\}$ and $\{\mathbf{s}(t)\}$, the trajectory of QSI $\{\mathbf{Q}(\omega, t)\}$ is uniquely determined. Let $\left\{\mathbf{p}^{E}(\omega, t)\right\}$ and $\left\{\mathbf{p}^{G}(\omega, t)\right\}$ be the sequences of the renewable power DTX control actions and grid power DTX control actions satisfying the structure in Lemma 1 for the given $\{\mathbf{p}(t)\}$, i.e., $p_{b}^{E}(\omega, t)=p_{b}(t) \mathbf{I}\left[E_{b}(\omega, t)>0\right]$ and $\left.p_{b}^{G}(\omega, t)=p_{b}(t) \mathbf{I}\left[E_{b}(\omega, t)=0\right]\right)$, where $\{\mathbf{E}(\omega, t)\}$ is the trajectory of ESI associated with $\left\{\mathbf{p}^{E}(\omega, t)\right\}$. Let $\left\{\mathbf{p}^{E \prime}(\omega, t)\right\}$ and $\left\{\mathbf{p}^{G \prime}(\omega, t)\right\}$ be any other sequences of feasible renewable power DTX control actions and grid power DTX control actions conditioned on $\{\mathbf{p}(\omega, t)\}$, i.e., $p_{b}^{E \prime}(\omega, t)+p_{b}^{G \prime}(\omega, t)=p_{b}(\omega, t)$, and $\left\{\mathbf{E}^{\prime}(\omega, t)\right\}$ be the trajectory of ESI associated with $\left\{\mathbf{p}^{E \prime}(\omega, t)\right\}$.

In the following, for each $b \in \mathcal{B}$, we shall show that for $\mathbf{E}^{\prime}(\omega, 1)=\mathbf{E}(\omega, 1)$, we have $\sum_{t=1}^{T} p_{b}^{G}(\omega, t) \leq$ $\sum_{t=1}^{T} p_{b}^{G \prime}(\omega, t)$. Let $\Delta p_{b}^{G}(\omega, T) \triangleq \sum_{t=1}^{T-1}\left(p_{b}^{G \prime}(\omega, t)-p_{b}^{G}(\omega, t)\right) \forall T \geq 2$ and $\Delta p_{b}^{G}(\omega, 1)=0$. Then, we have $\Delta p_{b}^{G}(\omega, t+1)=\Delta p_{b}^{G}(\omega, t)+\left(p_{b}^{G \prime}(\omega, t)-p_{b}^{G}(\omega, t)\right)$ for all $t \geq 1$. We shall prove $E_{b}(\omega, t)+\Delta p_{b}^{G}(t) \geq E_{b}^{\prime}(\omega, t)$ and $\Delta p_{b}^{G}(\omega, t) \geq 0$ for all $t \geq 1$ by induction. (In the following proof, we omit $\omega$ for notation simplicity.)

- Consider $t=1$. Since $E_{b}^{\prime}(1)=E_{b}(1)$ and $\Delta p_{b}^{G}(1)=0$ by the initial condition, we have $E_{b}(1)+\Delta p_{b}^{G}(1) \geq E_{b}^{\prime}(1)$ and $\Delta p_{b}^{G}(1) \geq 0$.

- For some $t \geq 1$, assume $E_{b}(t)+\Delta p_{b}^{G}(t) \geq E_{b}^{\prime}(t)$ and $\Delta p_{b}^{G}(t) \geq 0$. $E_{b}^{\prime}(t+1)=\min \left\{E_{b}^{\prime}(t)-\right.$ $\left.p^{E \prime}(t)+A_{b}^{E}(t), N_{E}\right\}$. We shall show the conclusions hold for $t+1$ by considering the following three cases. (1) When $E_{b}(t)>0$, we have $p_{b}^{E}(t)=p_{b}(t)$ and $p_{b}^{G}(t)=0$. Thus, we have $E_{b}(t+1)=\min \left\{E_{b}(t)-p(t)+A_{b}^{E}(t), N_{E}\right\}$ and $\Delta p_{b}^{G}(t+1)=\Delta p_{b}^{G}(t)+p_{b}^{G \prime}(t)-0 \geq 0$. In addition, since $p_{b}^{G \prime}(t)=p_{b}(t)-p_{b}^{E \prime}(t)$, we have $E_{b}(t+1)+\Delta p_{b}^{G}(t+1)=\min \left\{E_{b}(t)-\right.$ $\left.p_{b}(t)+A_{b}^{E}(t)+\Delta p_{b}^{G}(t)+p_{b}(t)-p_{b}^{E \prime}(t), N_{E}+\Delta p_{b}^{G}(t+1)\right\} \geq E_{b}^{\prime}(t+1)$. (2) When $E_{b}(t)=0$ and $E_{b}^{\prime}(t) \geq 1$, which implies $\Delta p_{b}^{G}(t) \geq 1$, we have $p_{b}^{E}(t)=0$ and $p_{b}^{G}(t)=p_{b}(t)$. Thus, we have $E_{b}(t+1)=\min \left\{E_{b}(t)+A_{b}^{E}(t), N_{E}\right\}$ and $\Delta p_{b}^{G}(t+1)=\Delta p_{b}^{G}(t)+p_{b}^{G \prime}(t)-p_{b}(t)=$ $\Delta p_{b}^{G}(t)-p_{b}^{E \prime}(t) \geq 1-1=0$, and hence, we have $E_{b}(t+1)+\Delta p_{b}^{G}(t+1)=\min \left\{E_{b}(t)+\right.$ 
$\left.A_{b}^{E}(t)+\Delta p_{b}^{G}(t)-p_{b}^{E \prime}(t), N_{E}+\Delta p_{b}^{G}(t+1)\right\} \geq E_{b}^{\prime}(t+1)$. (3) When $E_{b}(t)=0$ and $E_{b}^{\prime}(t)=0$, we have $p_{b}^{E}(t)=p_{b}^{E \prime}(t)=0$ and $p_{b}^{G}(t)=p_{b}^{G \prime}(t)=p_{b}(t)$. Thus, we have $E_{b}(t+1)=\min \{0+$ $\left.A_{b}^{E}(t), N_{E}\right\}, E_{b}^{\prime}(t+1)=\min \left\{0+A_{b}^{E}(t), N_{E}\right\}$ and $\Delta p_{b}^{G}(t+1)=\Delta p_{b}^{G}(t)+0 \geq 0$, and hence, $E_{b}(t+1)+\Delta p_{b}^{G}(t+1)=E_{b}^{\prime}(t+1)+\Delta p_{b}^{G}(t+1) \geq E_{b}^{\prime}(t+1)$.

Therefore, by induction, we can show $\Delta p_{b}^{G}(\omega, t) \geq 0$ for all $t$. Since the average delay costs per stage are the same, we have

$$
\frac{1}{T} \sum_{t=1}^{T}\left(\sum_{k} \beta_{k} f\left(Q_{k}(\omega, t)\right)+\sum_{b} \gamma_{b} p_{b}^{G}(\omega, t)\right) \leq \frac{1}{T} \sum_{t=1}^{T}\left(\sum_{k} \beta_{k} f\left(Q_{k}(\omega, t)\right)+\sum_{b} \gamma_{b} p_{b}^{G \prime}(\omega, t)\right)
$$

for any given $\{\mathbf{p}(t)\}$ and $\{\mathbf{s}(t)\}$ and $T$. By taking expectations over all sample paths, lim sup and optimizations over BS-DTX control and user selection policy space, we have $\min _{\pi} J_{\pi}^{(\boldsymbol{\beta}, \boldsymbol{\gamma})}(\chi(1)) \leq$ $\min _{\pi^{\prime}} J_{\pi^{\prime}}^{(\boldsymbol{\beta}, \boldsymbol{\gamma})}(\chi(1))$, where $\pi=\left\{\Omega^{1}, \Omega^{2}, \cdots\right\}$ with $\Omega^{t}$ satisfying the structure in Lemma 1 ,

\section{APPENDiX B: Proof of Lemma 2, COROllary 1 and Corollary 2}

Lemma 2. Based on Definition 2, we can transform the POMDP into the MDP with a tuple of the following four objects: state space $\mathcal{E} \times \mathcal{Q}$, action space $\mathcal{P} \times \mathcal{S}$ with partitioned architecture $\left\{\Omega^{t}(\mathbf{E}, \mathbf{Q})\right\}$ according to Definition 2 transition kernel $\operatorname{Pr}\left[\left(\mathbf{E}^{\prime}, \mathbf{Q}^{\prime}\right) \mid(\mathbf{E}, \mathbf{Q}), \Omega(\mathbf{E}, \mathbf{Q})\right]$, per-stage cost function $g((\mathbf{E}, \mathbf{Q}), \Omega(\mathbf{E}, \mathbf{Q}))$. Since the Weak Accessibility (WA) condition holds under our problem setup, by Proposition 4.2.3. in [11], the optimal average cost of the transformed MDP is the same for all initial states. In addition, by Proposition 4.1.3. and Proposition 4.1.4. in [11], we know that the solution $(\theta,\{V(\mathbf{E}, \mathbf{Q})\})$ to the Bellman equation in (10) exists. By Proposition 4.2.1. in [11], we can complete the proof.

Corollary [7: Define $\mathbb{Q}(\mathbf{E}, \mathbf{Q}, \mathbf{p}) \triangleq \min _{\Omega_{s}(\mathbf{E}, \mathbf{Q})}\left\{g\left((\mathbf{E}, \mathbf{Q}), \mathbf{p}, \Omega_{s}(\mathbf{E}, \mathbf{Q})\right)+\sum_{\left(\mathbf{E}^{\prime}, \mathbf{Q}^{\prime}\right)} \operatorname{Pr}\left[\left(\mathbf{E}^{\prime}, \mathbf{Q}^{\prime}\right) \mid(\mathbf{E}, \mathbf{Q}), \mathbf{p}, \Omega_{s}(\mathbf{E}\right.\right.$ $\theta$. Thus, we have $V(\mathbf{E}, \mathbf{Q})=\min _{\mathbf{p} \in \mathcal{P}} \mathbb{Q}(\mathbf{E}, \mathbf{Q}, \mathbf{p})$. Based on (10), we can obtain (12), which is in terms of BS-DTX control Q-factor $\{\mathbb{Q}(\mathbf{E}, \mathbf{Q}, \mathbf{p})\}$. From Lemma 2, we have the optimal BS-DTX control action given by (11).

Corollary 2]: Based on (10), we can obtain (14) [17]. For any $(\mathbf{E}, \mathbf{Q}) \in \mathcal{E} \times \mathcal{Q}$, as $\mathbf{p}^{*}=\Omega_{p}^{*}(\mathbf{E}, \mathbf{Q})$ can by obtained by (11), we can obtain $\Omega_{s}^{*}(\mathbf{E}, \mathbf{Q}, \mathbf{H})$ by solving the R.H.S. of (14) under $\mathbf{p}^{*}$ for any $\mathbf{A}^{E}$ and $\mathbf{A}^{Q}$ as follows:

$$
\begin{aligned}
& \min _{\Omega_{s}(\mathbf{E}, \mathbf{Q})}\left\{g\left((\mathbf{E}, \mathbf{Q}),\left(\mathbf{p}^{*}, \Omega_{s}(\mathbf{E}, \mathbf{Q})\right)\right)+\sum_{\left(\widetilde{\mathbf{E}^{\prime}}, \widetilde{\mathbf{Q}}^{\prime}\right)} \operatorname{Pr}\left[\left(\widetilde{\mathbf{E}}^{\prime}, \widetilde{\mathbf{Q}}^{\prime}\right) \mid(\mathbf{E}, \mathbf{Q}),\left(\mathbf{p}^{*}, \Omega_{s}(\mathbf{E}, \mathbf{Q})\right)\right] U\left(\widetilde{\mathbf{E}}^{\prime}, \widetilde{\mathbf{Q}}^{\prime}\right)\right\} \\
& \stackrel{(a)}{=} \min _{\Omega_{s}(\mathbf{E}, \mathbf{Q})}\left\{\sum_{\left(\widetilde{\mathbf{E}}^{\prime}, \widetilde{\mathbf{Q}}^{\prime}\right)} \mathbb{E}\left[\operatorname{Pr}\left[\left(\widetilde{\mathbf{E}}^{\prime}, \widetilde{\mathbf{Q}}^{\prime}\right) \mid \boldsymbol{\chi},\left(\mathbf{p}^{*}, \Omega_{s}(\boldsymbol{\chi})\right)\right] \mid(\mathbf{E}, \mathbf{Q})\right] U\left(\widetilde{\mathbf{E}}^{\prime}, \widetilde{\mathbf{Q}}^{\prime}\right)\right\}, \forall(\mathbf{E}, \mathbf{Q}) \in \mathcal{E} \times \mathcal{Q} \\
& \left.\stackrel{(b)}{\Leftrightarrow} \min _{\Omega_{s}(\boldsymbol{\chi})}\left\{\sum_{\left(\widetilde{\mathbf{E}}^{\prime}, \widetilde{\mathbf{Q}}^{\prime}\right)} \operatorname{Pr}\left[\left(\widetilde{\mathbf{E}}^{\prime}, \widetilde{\mathbf{Q}}^{\prime}\right) \mid \chi,\left(\mathbf{p}^{*}, \Omega_{s}(\boldsymbol{\chi})\right)\right] \mid(\mathbf{E}, \mathbf{Q})\right] U\left(\widetilde{\mathbf{E}}^{\prime}, \widetilde{\mathbf{Q}}^{\prime}\right)\right\}, \forall \boldsymbol{\chi} \in \mathcal{X} \\
& \stackrel{(c)}{=} \min _{\mathbf{s} \in \mathcal{S}\left(\mathbf{p}^{*}\right)}\left\{\sum_{\mathbf{d} \in \mathcal{D}}\left(\prod_{k \in \mathcal{K}}\left(1-d_{k}-(-1)^{d_{k}} \operatorname{Pr}\left[\rho_{k}\left(\mathbf{H}, \mathbf{p}^{*}, \mathbf{s}\right) \geq \delta_{k}\right]\right) U\left(\left[\mathbf{E}-\mathbf{p}^{*}\right]^{+},[\mathbf{Q}-\mathbf{d}]^{+}\right)\right)\right\}
\end{aligned}
$$


where (a) is due to the definition of $g(\cdot, \cdot)$ and $\left.\operatorname{Pr}\left[\left(\widetilde{\mathbf{E}}^{\prime}, \widetilde{\mathbf{Q}}^{\prime}\right) \mid(\mathbf{E}, \mathbf{Q}), \Omega(\mathbf{E}, \mathbf{Q})\right)\right]$, (b) is due to Definition 2 and (c) is due to Assumptions 3 and 4 as well as $E_{b}-p_{b}^{E *}=E_{b}-p_{b}^{*} \mathbf{I}\left[E_{b}>0\right]=\left[E_{b}-p_{b}^{*}\right]^{+}$.

\section{ApPendix C: Proof of Lemma 3}

We shall prove the additive property w.r.t. the potential function. Following the proofs of Corollary 1 and Corollary 2, the additive property can be easily extended to the Q-factor and the post-decision potential function. Let $\hat{\theta}$ and $\hat{V}(\mathbf{E}, \mathbf{Q})$ be the average cost and the potential function under $\hat{\Omega}$. Then, we have the following Bellman equation in terms of $(\hat{\theta},\{\hat{V}(\mathbf{E}, \mathbf{Q})\})$ :

$$
\hat{\theta}+\hat{V}(\mathbf{E}, \mathbf{Q})=\mathbb{E}^{\hat{\Omega}_{p}}[\hat{g}((\mathbf{E}, \mathbf{Q}), \mathbf{p})]+\sum_{\left(\mathbf{E}^{\prime}, \mathbf{Q}^{\prime}\right)} \mathbb{E}^{\hat{\Omega}_{p}}\left[\hat{\operatorname{Pr}}\left[\left(\mathbf{E}^{\prime}, \mathbf{Q}^{\prime}\right) \mid(\mathbf{E}, \mathbf{Q}), \mathbf{p}\right]\right] \hat{V}\left(\mathbf{E}^{\prime}, \mathbf{Q}^{\prime}\right)
$$

where $\hat{g}((\mathbf{E}, \mathbf{Q}), \mathbf{p})=g((\mathbf{E}, \mathbf{Q}), \mathbf{p})$ and $\hat{\operatorname{Pr}}\left[\left(\mathbf{E}^{\prime}, \mathbf{Q}^{\prime}\right) \mid(\mathbf{E}, \mathbf{Q}), \mathbf{p}\right]=\mathbb{E}\left[\mathbb{E}^{\hat{\Omega}_{s}}\left[\operatorname{Pr}\left[\left(\mathbf{E}^{\prime}, \mathbf{Q}^{\prime}\right) \mid(\mathbf{E}, \mathbf{Q}, \mathbf{H}), \mathbf{p}, \mathbf{s}\right] \mid \mathbf{p}, \mathbf{H}\right] \mid \mathbf{p}\right]$. Let $\hat{\theta}_{k}$ and $\hat{V}_{k}(\mathbf{E}, \mathbf{Q})$ be the per-flow average cost and potential function under $\hat{\Omega}$. Then, we have the following per-flow fixed point equation in terms of $\left(\hat{\theta}_{k},\left\{\hat{V}_{k}\left(E_{b}, Q_{k}\right)\right\}\right)$ :

$$
\hat{\theta}_{k}+\hat{V}_{k}\left(E_{b}, Q_{k}\right)=\mathbb{E}^{\hat{\Omega}_{p}}\left[\hat{g}_{k}\left(\left(E_{b}, Q_{k}\right), p_{b}\right)\right]+\sum_{\left(E_{b}^{\prime}, Q_{k}^{\prime}\right)} \mathbb{E}^{\hat{\Omega}_{p}}\left[\hat{\operatorname{Pr}}\left[\left(E_{b}^{\prime}, Q_{k}^{\prime}\right) \mid\left(E_{b}, Q_{k}\right), \mathbf{p}\right]\right] \hat{V}_{k}\left(E_{b}^{\prime}, Q_{k}^{\prime}\right)
$$

Under $\hat{\Omega}$, the induced Markov chain has a single recurrent class. Therefore, the solutions to (30) and (31) exist, respectively. First, we have $\mathbb{E}^{\hat{\Omega}_{p}}[\hat{g}((\mathbf{E}, \mathbf{Q}), \mathbf{p})]=\sum_{b \in \mathcal{B}} \sum_{k \in \mathcal{K}_{b}} \mathbb{E}^{\hat{\Omega}_{p}}\left[\hat{g}_{k}\left(\left(E_{b}, Q_{k}\right), p_{b}\right)\right]$. Second, by the relationship between the joint distribution and the marginal distribution, we have $\sum_{\left(\mathbf{E}^{\prime}, \mathbf{Q}^{\prime}\right)} \hat{\operatorname{Pr}}\left[\left(\mathbf{E}^{\prime}, \mathbf{Q}^{\prime}\right) \mid(\mathbf{E}, \mathbf{Q}), \mathbf{p}\right]=\sum_{\left(E_{b}^{\prime}, Q_{k}^{\prime}\right)} \hat{\operatorname{Pr}}\left[\left(E_{b}^{\prime}, Q_{k}^{\prime}\right) \mid(\mathbf{E}, \mathbf{Q}), \mathbf{p}\right]=\sum_{\left(E_{b}^{\prime}, Q_{k}^{\prime}\right)} \hat{\operatorname{Pr}}\left[\left(E_{b}^{\prime}, Q_{k}^{\prime}\right) \mid\left(E_{b}, Q_{k}\right), \mathbf{p}\right]$. Therefore, substitute $\hat{\theta}=\sum_{k \in \mathcal{K}} \hat{\theta}_{k}$ and $\hat{V}(\mathbf{E}, \mathbf{Q})=\sum_{b \in \mathcal{B}} \sum_{k \in \mathcal{K}_{b}} \hat{V}_{k}\left(E_{b}, Q_{k}\right)$ into (30), we can see that the equality holds. Therefore, we complete the proof.

\section{APPENDix D: Proof of Lemma4}

Using the approximation in (19) and (29), we have

$$
\begin{aligned}
& \min _{\mathbf{s} \in \mathcal{S}\left(\hat{\mathbf{p}}^{*}\right)}\left\{\sum_{\mathbf{d} \in \mathcal{D}}\left(\prod_{k \in \mathcal{K}}\left(1-d_{k}-(-1)^{d_{k}} \operatorname{Pr}\left[\rho_{k} \geq \delta_{k}\right]\right)\left(\sum_{n} \sum_{k \in \mathcal{K}_{n}} \hat{U}_{k}\left(\left[E_{b}-\hat{p}_{b}^{*}\right]^{+},\left[Q_{k}-d_{k}\right]^{+}\right)\right)\right)\right\} \\
& =\min _{\mathbf{s} \in \mathcal{S}\left(\hat{\mathbf{p}}^{*}\right)}\left\{\sum_{n} \sum_{k \in \mathcal{K}_{n}} \sum_{d_{k} \in \mathcal{D}}\left(1-d_{k}-(-1)^{d_{k}} \operatorname{Pr}\left[\rho_{k} \geq \delta_{k}\right]\right) \hat{U}_{k}\left(\left[E_{b}-\hat{p}_{b}^{*}\right]^{+},\left[Q_{k}-d_{k}\right]^{+}\right)\right\} \\
& \Leftrightarrow \min _{\mathbf{s}_{n} \in \mathcal{S}_{n}\left(\hat{\mathbf{p}}_{n}^{*}\right)} \sum_{k \in \mathcal{K}_{n}} \sum_{d_{k} \in \mathcal{D}_{k}}\left(1-d_{k}-(-1)^{d_{k}} \operatorname{Pr}\left[\rho_{k} \geq \delta_{k}\right]\right) \hat{U}_{k}\left(\left[E_{b}-\hat{p}_{b}^{*}\right]^{+},\left[Q_{k}-d_{k}\right]^{+}\right), \forall n \\
& =\min _{\mathbf{s}_{n} \in \mathcal{S}_{n}\left(\hat{\mathbf{p}}_{n}^{*}\right)} \sum_{k \in \mathcal{K}_{n}}\left(\left(1-s_{k}\right) \hat{U}_{k}\left(\left[E_{b}-\hat{p}_{b}^{*}\right]^{+}, Q_{k}\right)\right. \\
& \left.\quad+s_{k}\left(\operatorname{Pr}\left[\rho_{k} \geq \delta_{k}\right] \hat{U}_{k}\left(\left[E_{b}-\hat{p}_{b}^{*}\right]^{+},\left[Q_{k}-1\right]^{+}\right)+\left(1-\operatorname{Pr}\left[\rho_{k} \geq \delta_{k}\right]\right)\right) \hat{U}_{k}\left(\left[E_{b}-\hat{p}_{b}^{*}\right]^{+}, Q_{k}\right)\right) \\
& \Leftrightarrow \\
& \qquad \min _{\mathbf{s}_{n} \in \mathcal{S}_{n}\left(\hat{\mathbf{p}}_{n}^{*}\right)} \sum_{k \in \mathcal{K}_{n}} s_{k} \operatorname{Pr}\left[\rho_{k} \geq \delta_{k}\right]\left(\hat{U}_{k}\left(\left[E_{b}-\hat{p}_{b}^{*}\right]^{+},\left[Q_{k}-1\right]^{+}\right)-\hat{U}_{k}\left(\left[E_{b}-\hat{p}_{b}^{*}\right]^{+}, Q_{k}\right)\right), \forall n
\end{aligned}
$$




\section{APPendix E: Proof of TheOrem 1}

Under the assumptions 3 and 4 as well as $\hat{\Omega}$ in Definition 3 , Markov chain $\{(\mathbf{E}(t), \mathbf{Q}(t))\}$ has a single recurrent class (and possibly some transient states). Thus, $\hat{\Omega}$ is a unchain policy. In addition, it is obvious that $\hat{\Omega}^{*} \neq \hat{\Omega}$. Therefore, the conditions of Proposition 4.4.2 in [11] are satisfied expect for the assumption that $\hat{\Omega}^{*}$ is a unchain policy. We shall modify the proof of Proposition 4.4.2 to incorporate a general $\hat{\Omega}^{*}$ as follows. We adopt the same notations as Proposition 4.4.2. ( $\mu$ can be treated as $\hat{\Omega}$ and $\bar{\mu}$ can be treated as $\left.\hat{\Omega}^{*}\right)$. Let $\left(\bar{\lambda}, \mathbf{h}_{\bar{\mu}}\right)$ be the gain-bias pair of a general $\bar{\mu}$. Thus, by Proposition 4.1.9, $\left(\bar{\lambda}, \mathbf{h}_{\bar{\mu}}\right)$ satisfies $\bar{\lambda}=\bar{P} \bar{\lambda}$ and $\bar{\lambda}+\mathbf{h}_{\bar{\mu}}=\mathbf{T}_{\bar{\mu}} \mathbf{h}_{\bar{\mu}}$. However, let $\left(\lambda, \mathbf{h}_{\mu}\right)$ be the gain-bias pair of a unchain $\mu$, which satisfies $\lambda \mathbf{e}+\mathbf{h}_{\mu}=\mathbf{T}_{\mu} \mathbf{h}_{\mu}$. Since $\operatorname{Pr}\left[\left(\mathbf{E}^{\prime}, \mathbf{Q}^{\prime}\right) \mid(\mathbf{E}, \mathbf{Q}),(\mathbf{p}, \mathbf{s})\right] \neq \operatorname{Pr}\left[\left(\mathbf{E}^{\prime}, \mathbf{Q}^{\prime}\right) \mid(\mathbf{E}, \mathbf{Q}),\left(\mathbf{p}^{\prime}, \mathbf{s}^{\prime}\right)\right]$, there is strict performance improvement under $\hat{\Omega}^{*}$ over $\hat{\Omega}$. Thus, we have a stronger result than (4.97), i.e. $\delta(i)>0 \forall i$. To incorporate a general $\bar{\mu}$, we have $\boldsymbol{\delta}=(\lambda \mathbf{e}-\overline{\boldsymbol{\lambda}})+(\mathbf{I}-\overline{\mathbf{P}}) \boldsymbol{\Delta}$ instead of (4.98). Since $\overline{\boldsymbol{\lambda}}=\bar{P} \overline{\boldsymbol{\lambda}}$, we have $\sum_{k=0}^{N-1} \overline{\mathbf{P}}^{k} \boldsymbol{\delta}=N(\lambda \mathbf{e}-\overline{\boldsymbol{\lambda}})+\left(\mathbf{I}-\overline{\mathbf{P}}^{N}\right) \boldsymbol{\Delta}$ in stead of (4.99), which implies $\overline{\mathbf{P}}^{*} \boldsymbol{\delta}=\lambda \mathbf{e}-\overline{\boldsymbol{\lambda}}$ instead of (4.100). Since $\delta(i)>0 \forall i$, we have $\lambda>\overline{\boldsymbol{\lambda}}(i) \forall i$. In other words, we can show $\hat{\theta}^{*}(\mathbf{E}, \mathbf{Q})<\hat{\theta}$ for all $(\mathbf{E}, \mathbf{Q}) \in \mathcal{E} \times \mathcal{Q}$.

\section{APPENDix F: Proof of LeMma 5}

Note that the update equations in (21) and (22) can be treated as the synchronous stochastic versions of the synchronous relative value iterations (RVI) [11] for the Markov chains $\left\{\left(E_{b}(t), Q_{k}(t), \mathbf{p}(t)\right)\right\}$ $\left(\left\{\left(E_{b}(t), Q_{k}(t)\right)\right\}\right)$ with the policy space containing only one policy $\hat{\Omega}[11]$. Under $\hat{\Omega}$ defined in Definition 3, the two Markov chains have a single recurrent class (and possibly some transient states). Therefore, the condition of Lemma 2 in [24] holds, according to the explanation for the conditions of Proposition 4.3.2 in [11]. Following the proof of Lemma 2 in [24], which is a modified version of the proof for Proposition 4.3.2 in [11], we can prove Lemma 5. We omit the details here due to page limit.

\section{APPENDiX G: PROOF OF LEMMA 6}

From the conditional coverage probability (conditioned on the nearest BS being at a distance $r_{1}$ from the randomly chosen MS) for cellular networks without BS coordination obtained in [12], we have the conditional successful packets transmission probability of the randomly chosen MS given by $p_{s}\left(r_{1}, \lambda^{\prime}\right) \leq \exp \left(-C_{1} \lambda^{\prime}-\frac{N_{0}}{P} \delta r_{1}^{\alpha}\right)$, where $\lambda^{\prime}$ is the density of the homogeneous PPP $\Phi^{\prime}$ used to model the locations of active BSs and the inequality is due to $\eta(x, \alpha)=\int_{x^{-\frac{2}{\alpha}}}^{\infty} \frac{1}{1+u^{\frac{\alpha}{2}}} d u \leq \int_{x^{-\frac{2}{\alpha}}}^{\infty} u^{-\frac{\alpha}{2}} d u=$ $\frac{2 x^{1-\frac{2}{\alpha}}}{\alpha-2} \triangleq \tilde{\eta}(x, \alpha)$.

Next, we shall show sufficiency by proving that (25) guarantees stability in a parallel dominant network, in which dummy packets are transmitted when a data queue is empty. Sending dummy packets is only aimed to cause interference to the other MSs and not counted as an actual packet 
transmission. The dominant system stochastically dominates the original system in the sense that the queue sizes and grid power costs in that system are necessarily not smaller (bigger) than those in the original system. Therefore, the stability conditions obtained for the dominant systems are sufficient for the stability of the original system. In the dominant system, since $\operatorname{Pr}\left[p_{b}=1\right]=p_{t x}$, we have $\lambda^{\prime}=p_{t x} \lambda$. Therefore, the service rate of the randomly chosen MS is $\mu\left(p_{t x}, \lambda\right)=p_{t x} p_{s}\left(r_{1}, \lambda^{\prime}\right)$. By Loynes' Theorem, the queue of the randomly chosen MS is stable if $\lambda^{Q}<\mu\left(p_{t x}, \lambda\right)$. Thus, we complete the proof for (25). Note that $E_{b}$ is decoupled from $Q_{k}$ and forms a discrete-time $M / M / 1 / N_{E}$ system with arrival rate $\lambda^{E}$ and departure rate $p_{t x}$. By queueing theory, we have $\operatorname{Pr}\left[E_{b}>\right.$ $0]=f\left(p_{t x}, N_{E}\right)$ [21]. Thus, we can prove the average grid power cost in the dominant system is $\overline{p_{\max }^{G}}\left(p_{t x}, N_{E}\right)=\left(1-f\left(p_{t x}, N_{E}\right)\right) p_{t x}$.

\section{APPENDix H: ProOf OF LEMMa 7}

In the following proof, we shall focus on the derivation of the conditional successful packet transmission probability $p_{s}\left(r_{1}, \lambda, \lambda^{\prime}, N_{t}\right)$. The remaining proof is similar to that in the proof of Lemma 6. Let $b_{i}$ denote the $i$-th nearest BS among all the BSs (including those are on and off) to the randomly chosen MS $k_{0}$, where $i=1, \cdots, N_{t}$. Thus, $b_{1}$ is the BS of MS $k_{0}$. By forming a cluster $\mathcal{B}_{0}=\left\{b_{1}, \cdots, b_{N_{t}}\right\} \subset \Phi$, MS $k_{0}$ can achieve the highest $p_{s}\left(r_{1}, \lambda, \lambda^{\prime}, N_{t}\right)$. We shall calculate $p_{s}\left(r_{1}, \lambda, \lambda^{\prime}, N_{t}\right)$ under the favorable cluster $\mathcal{B}_{0} \subset \Phi$. Let $R_{1}$ and $R_{N_{t}}$ denote the distance between BS $b_{1}$ and MS $k_{0}$ as well as the distance between BS $b_{N_{t}}$ and MS $k_{0}$. First, we shall derive the conditional p.d.f. $f_{R_{N_{t}} \mid R_{1}}\left(r_{N_{t}} \mid r_{1}\right)$ and the conditional expectation $E\left[R_{N_{t}}^{2-\alpha} \mid R_{1}=r_{1}\right]$. If $r_{B} \leq r_{1}$, we have $\operatorname{Pr}\left[R_{N_{t}}>r_{N_{t}} \mid R_{1}=r_{1}\right]=1 \Rightarrow f_{R_{N_{t}} \mid R_{1}}\left(r_{N_{t}} \mid r_{1}\right)=0$. It remains to consider $r_{N_{t}}>r_{1}$. Let $\mathcal{B}_{2}(0, r)$ denote the 2 -dim ball centered in the origin with radius $r$. Following similar techniques in [25], we have, for $r_{N_{t}}>r_{1}$,

$$
\begin{aligned}
& y=\operatorname{Pr}\left[R_{N_{t}}>r_{N_{t}} \mid R_{1}=r_{1}\right]=\operatorname{Pr}\left[0,1, \cdots, N_{t}-2 \text { BSs in } \mathcal{B}_{2}\left(0, r_{N_{t}}\right)-\mathcal{B}_{2}\left(0, r_{1}\right)\right] \\
= & \sum_{i=0}^{N_{t}-2} \frac{\left(\lambda \pi\left(r_{N_{t}}^{2}-r_{1}^{2}\right)\right)^{i}}{i !} \exp \left(-\lambda \pi\left(r_{N_{t}}^{2}-r_{1}^{2}\right)\right) \\
\Rightarrow & f_{R_{N_{t}} \mid R_{1}}\left(r_{N_{t}} \mid r_{1}\right)=-\frac{d y}{d r_{N_{t}}}=2 \lambda \pi r_{b} \exp \left(-\lambda \pi\left(r_{N_{t}}^{2}-r_{1}^{2}\right)\right) \sum_{i=0}^{N_{t}-2} \frac{\left(\lambda \pi\left(r_{N_{t}}^{2}-r_{1}^{2}\right)\right)^{i}}{i !} \\
& \quad-\exp \left(-\lambda \pi\left(r_{N_{t}}^{2}-r_{1}^{2}\right)\right) \sum_{i=1}^{N_{t}-2} \frac{\lambda \pi i\left(\lambda \pi\left(r_{N_{t}}^{2}-r_{1}^{2}\right)\right)^{i-1} 2 r_{N_{t}}}{i !} \\
= & 2 \lambda \pi r_{N_{t}} \exp \left(-\lambda \pi\left(r_{N_{t}}^{2}-r_{1}^{2}\right)\right) \frac{\left(\lambda \pi\left(r_{N_{t}}^{2}-r_{1}^{2}\right)\right)^{N_{t}-2}}{\left(N_{t}-2\right) !}, \quad r_{N_{t}}>r_{1} \\
\Rightarrow & \mathbb{E}\left[R_{N_{t}}^{2-\alpha} \mid R_{1}=r 1\right]=\int_{0}^{\infty} r_{N_{t}}^{2-\alpha} f_{R_{N_{t}} \mid R_{1}}\left(r_{N_{t}} \mid r_{1}\right) d r_{N_{t}} \\
= & \frac{(\lambda \pi)^{N_{t}-1}}{\left(N_{t}-2\right) !} \int_{r_{1}}^{\infty}\left(r_{N_{t}}^{2}\right)^{1-\frac{\alpha}{2}} \exp \left(-\lambda \pi\left(r_{N_{t}}^{2}-r_{1}^{2}\right)\right)\left(r_{N_{t}}^{2}-r_{1}^{2}\right)^{N_{t}-2} d\left(r_{N_{t}}^{2}-r_{1}^{2}\right)
\end{aligned}
$$




$$
\stackrel{(a)}{\leq} \frac{(\lambda \pi)^{N_{t}-1}}{\left(N_{t}-2\right) !} \int_{0}^{\infty} u^{1-\frac{\alpha}{2}} \exp (-\lambda \pi u) u^{N_{t}-2} d u=(-\lambda \pi)^{\frac{\alpha}{2}-1} \frac{\Gamma\left(N_{t}-\frac{\alpha}{2}\right)}{\Gamma\left(N_{t}-1\right)}, \quad N_{t}>\frac{\alpha}{2}
$$

where (a) is due to $\alpha>2$ and the change of variables $u=r_{N_{t}}^{2}-r_{1}^{2}$. (a) is tight for small $r_{1}$. In addition, $\mathbb{E}\left[R_{N_{t}}^{2-\alpha} \mid R_{1}=r 1\right] \leq r_{1}^{2-\alpha}$.

Next, we shall calculate $p_{s}\left(r_{1}, \lambda, \lambda^{\prime}, N_{t}\right)$. Note that the interference to MS $k_{0}$ comes from the active BSs in $\Phi^{\prime}-\mathcal{B}_{0} \bigcap \Phi^{\prime}$. In addition, the signal power $G_{1}$ and interference power $G_{b}$ (from the active BS $b \in \Phi^{\prime}-\mathcal{B}_{0} \bigcap \Phi^{\prime}$ ) due to small scale fading are exponentially distributed with mean 1 [26]. Let $I_{R_{N_{t}}}$ denote the interference, which is a function of random variable $R_{B}$. Therefore, we have

$$
\begin{aligned}
& p_{s}\left(r_{1}, \lambda, \lambda^{\prime}, N_{t}\right)=\operatorname{Pr}\left[S I N R \geq \delta \mid R_{1}=r_{1}\right]=\operatorname{Pr}\left[\frac{P G_{1} r_{1}^{-\alpha}}{N_{0}+I_{R_{N_{t}}}} \geq \delta\right] \\
= & \mathbb{E}_{R_{N_{t}}}\left[\mathbb{E}_{I_{R_{N_{t}}}}\left[\operatorname{Pr}\left[G_{1} \geq \frac{1}{P} \delta r_{1}^{\alpha}\left(N_{0}+I_{R_{N_{t}}}\right) \mid I_{R_{N_{t}}}\right]\right]\right]=\mathbb{E}_{R_{N_{t}}}\left[\mathbb{E}_{I_{R_{N_{t}}}}\left[\exp \left(-\frac{1}{P} \delta r_{1}^{\alpha}\left(N_{0}+I_{R_{N_{t}}}\right)\right)\right]\right] \\
= & \exp \left(-\frac{N_{0}}{P} \delta r_{1}^{\alpha}\right) \mathbb{E}_{R_{N_{t}}}\left[\mathbb{E}_{I_{R_{N_{t}}}}\left[\exp \left(-\frac{1}{P} \delta r_{1}^{\alpha} I_{R_{N_{t}}}\right)\right]\right]
\end{aligned}
$$

Let $s=-\frac{1}{P} \delta r_{1}^{\alpha}$ and $R_{b}$ denote the distance between BS $b \in \Phi^{\prime}-\mathcal{B}_{0} \bigcap \Phi^{\prime}$ and MS $k_{0}$, we have

$$
\begin{aligned}
& \mathbb{E}_{I_{R_{N_{t}}}}\left[\exp \left(-s I_{R_{N_{t}}}\right)\right]=\mathbb{E}_{\Phi^{\prime},\left\{G_{b}\right\}}\left[\exp \left(-s \sum_{b \in \Phi^{\prime}-\mathcal{B}_{0} \cap \Phi^{\prime}} P G_{b} R_{b}^{-\alpha}\right)\right] \\
= & \mathbb{E}_{\Phi^{\prime},\left\{G_{b}\right\}}\left[\prod_{b \in \Phi^{\prime}-\mathcal{B}_{0} \cap \Phi^{\prime}} \exp \left(-s P G_{b} R_{b}^{-\alpha}\right)\right] \\
= & \mathbb{E}_{\Phi^{\prime}}\left[\prod_{b \in \Phi^{\prime}-\mathcal{B}_{0} \cap \Phi^{\prime}} \mathbb{E}_{\left\{G_{b}\right\}}\left[\exp \left(-s P G_{b} R_{b}^{-\alpha}\right)\right]\right]=\mathbb{E}_{\Phi^{\prime}}\left[\prod_{b \in \Phi^{\prime}-\mathcal{B}_{0} \cap \Phi^{\prime}} \frac{1}{1+s P G_{b} R_{b}^{-\alpha}}\right] \\
= & \exp \left(-2 \lambda^{\prime} \pi \int_{R_{N_{t}}}\left(1-\frac{1}{1+s P G_{b} R_{b}^{-\alpha}}\right)\right) v d v \stackrel{(a)}{=} \exp \left(-2 \lambda^{\prime} \pi \int_{r_{1} \frac{R_{N_{t}}}{r_{1}}} \frac{1+\left(\frac{v}{r_{1} \delta^{1 / \alpha}}\right)^{-\alpha}}{1} d v\right) \\
& \stackrel{(b)}{=} \exp \left(-\lambda^{\prime} \pi r_{1}^{2} \delta^{\frac{2}{\alpha}} \int_{\delta^{-\frac{2}{\alpha}}\left(\frac{R_{N_{t}}}{r_{1}}\right)^{2}} \frac{1+u^{\frac{\alpha}{2}}}{1} v d v\right)=\exp \left(-\lambda^{\prime} \pi r_{1}^{2} \delta^{\frac{2}{\alpha}} \eta\left(\delta\left(\frac{r_{1}}{R_{N_{t}}}\right)^{\alpha}, \alpha\right)\right) \\
\Rightarrow & \mathbb{E}_{R_{N_{t}}}\left[\mathbb{E}_{I_{R_{N_{t}}}}\left[\exp \left(-\frac{1}{P} \delta r_{1}^{\alpha} I_{R_{N_{t}}}\right)\right]\right] \stackrel{(c)}{\geq} \exp \left(-\lambda^{\prime} \pi r_{1}^{2} \delta^{\frac{2}{\alpha}} E_{R_{N_{t}}}\left[\eta\left(\delta\left(\frac{r_{1}}{R_{N_{t}}}\right)^{\alpha}, \alpha\right)\right]\right) \\
(d) & \exp \left(-\lambda^{\prime} \pi r_{1}^{2} \delta^{\frac{2}{\alpha}} E_{R_{N_{t}}}\left[\tilde{\eta}\left(\delta\left(\frac{r_{1}}{R_{N_{t}}}\right)^{\alpha}, \alpha\right)\right]\right)=\exp \left(-\lambda^{\prime} \frac{2}{\alpha-2} \pi r_{1}^{2} \delta r_{1}^{\alpha-2} E_{R_{N_{t}}}\left[\left(R_{N_{t}}\right)^{2-\alpha} \mid R_{1}=r_{1}\right]\right) \\
\geq & \stackrel{(e)}{\geq} \exp \left(-C_{N_{t}} \lambda^{\prime}\right)
\end{aligned}
$$

where (a) is due to plugging in $s=-\frac{1}{P} \delta r_{1}^{\alpha}$, (b) is due to the change of variables $u=\left(\frac{v}{r_{1} \delta^{1 / \alpha}}\right)^{-\alpha}$, (c) is due to the convexity of the exponential function, (d) is due to $\eta(x, \alpha)=\int_{x^{-\frac{2}{\alpha}}}^{\infty} \frac{1}{1+u^{\frac{\alpha}{2}}} d u \leq$ $\int_{x^{-\frac{2}{\alpha}}}^{\infty} u^{-\frac{\alpha}{2}} d u=\frac{2 x^{1-\frac{2}{\alpha}}}{\alpha-2} \triangleq \tilde{\eta}(x, \alpha)$, (e) is due to inequality (33) and $\mathbb{E}\left[R_{N_{t}}^{2-\alpha} \mid R_{1}=r 1\right] \leq r_{1}^{2-\alpha}$. Substituting (35) into (34), we have $p_{s}\left(r_{1}, \lambda, \lambda^{\prime}, N_{t}\right) \geq \exp \left(-C_{N_{t}} \lambda^{\prime}-\frac{N_{0}}{P} \delta r_{1}^{\alpha}\right)$. Since $\lambda^{\prime}=p_{t x} \lambda$, we can prove (27). 


\section{APPENDIX I: Proof of Theorem 2}

$$
\begin{aligned}
& \frac{d \lambda_{\max }^{Q}}{d p_{t x}}=\left(1-C_{N_{t}} \lambda p_{t x} \lambda\right) \exp \left(-C_{N_{t}} p_{t x} \lambda-\frac{N_{0}}{P} \delta r_{1}^{\alpha}\right) \begin{cases}>0, & p_{t x}<\frac{1}{C_{N_{t}} \lambda} \\
\leq 0, & p_{t x} \geq \frac{1}{C_{N_{t}} \lambda}\end{cases} \\
& \frac{\partial \overline{p_{\max }^{G}}\left(p_{t x}, N_{E}\right)}{\partial p_{t x}}=1-\frac{\partial f\left(p_{t x}, N_{E}\right)}{\partial p_{t x}}+\left(1-f\left(p_{t x}, N_{E}\right)\right)>0, \quad \forall N_{E}>0
\end{aligned}
$$

where the last inequality is due to $\frac{\partial f\left(p_{t x}, N_{E}\right)}{\partial p_{t x}}<0$ and $f\left(p_{t x}, N_{E}\right) \leq 1$. In addition, $p_{t x} \in[0,1]$. Therefore, we can easily obtain $p_{t x}^{*}\left(N_{E}, N_{t}\right)$. Next, we shall prove the property of $\lambda_{\max }^{Q *}\left(N_{E}, N_{t}\right)$ w.r.t. $N_{E}$. It is obvious that $x^{*}\left(N_{E}\right)$ increases with $N_{E}$. If $x^{*}\left(N_{E}\right)<\min \left\{1, \frac{1}{C_{N_{t}} \lambda}\right\}, p_{t x}^{*}\left(N_{E}\right)=x^{*}\left(N_{E}\right)$ and $\left.\frac{d \lambda_{\max }^{Q}}{d p_{t x}}\right|_{p_{t x}=p_{t x}^{*}\left(N_{E}, N_{t}\right)}>0$. Thus, $\lambda_{\max }^{Q *}\left(N_{E}, N_{t}\right)$ is increasing in $N_{E}$. If $x^{*}\left(N_{E}\right) \geq \min \left\{1, \frac{1}{C_{N_{t}} \lambda}\right\}$, $p_{t x}^{*}\left(N_{E}\right)=\min \left\{1, \frac{1}{C_{N_{t}} \lambda}\right\} \cdot \lambda_{\max }^{Q *}\left(N_{E}, N_{t}\right)$ is a constant for all $N_{E}$. Finally, we shall show the property of $\lambda_{\max }^{Q *}\left(N_{E}, N_{t}\right)$ w.r.t. $N_{t}$ for any given $N_{E}>0$. It can be easily verified that $C_{N_{t}}$ is decreasing in $N_{t}$. Thus, when $p_{t x}^{*}\left(N_{E}, N_{t}\right)=x^{*}\left(N_{E}\right)$ or 1 , we have that $\lambda_{\max }^{Q *}\left(N_{E}, N_{t}\right)=$ $p_{t x}^{*}\left(N_{E}\right) \exp \left(-C_{N_{t}} p_{t x}^{*}\left(N_{E}\right) \lambda-\frac{N_{0}}{P} \delta r_{1}^{\alpha}\right)$ is increasing in $N_{t}$. When $p_{t x}^{*}\left(N_{E}, N_{t}\right)=\frac{1}{C_{N_{t}} \lambda}$, we have that $\lambda_{\max }^{Q *}\left(N_{E}, N_{t}\right)=C_{N_{t}} \lambda \exp \left(-1-\frac{N_{0}}{P} \delta r_{1}^{\alpha}\right)$ is increasing in $N_{t}$.

\section{REFERENCES}

[1] D. Gesbert, S. Hanly, H. Huang, S. Shamai Shitz, O. Simeone, and W. Yu, "Multi-cell MIMO cooperative networks: A new look at interference," IEEE J. Select. Areas Commun., vol. 28, no. 9, pp. 1380-1408, Dec. 2010.

[2] H. Dahrouj and W. Yu, "Coordinated beamforming for the multicell multi-antenna wireless system," IEEE Trans. Wireless Commun., vol. 9, no. 5, p. 17481759, May 2010.

[3] J. Zhang, R. Chen, J. G. Andrews, A. Ghosh, and R. W. Heath, Jr., "Networked MIMO with clustered linear precoding," IEEE Trans. Wireless Commun., vol. 8, pp. 1910-1921, Apr. 2009.

[4] K. Huang and J. G. Andrews, "Characterizing multi-cell cooperation via the outage-probability exponent," in Proc. of IEEE Int. Conf. on Commun. (ICC), Ottawa, Canada, Jun 2012.

[5] J. Zhang, R. Chen, J. G. Andrews, and R. W. Heath, Jr., "Coordinated multi-cell MIMO systems with cellular block diagonalization," in Proc. of Asilomar Conference on Signals, Systems and Computers (ACSSC), Pacific Grove, CA, USA, Nov. 2007, pp. 1669-1673.

[6] F. Boccardi and H. Huang, "Limited downlink network coordination in cellular networks," in IEEE International Symposium on Personal, Indoor and Mobile Radio Communications (PIMRC), Athens, Greece, Sept. 2007, pp. 1-5.

[7] S. Brueck, L. Zhao, J. Giese, and M. A. Amin, "Centralized scheduling for joint transmission coordinated multipoint in LTE-advanced," in Proc. of International ITG Workshop on Smart Antennas (WSA), Bremen, Feb. 2010, pp. 177-184.

[8] L. Huang and M. J. Neely, "Utility optimal scheduling in energy harvesting networks," in Proc. of Mobihoc, Paris, France, May 2011.

[9] M. Gatzianas, L. Georgiadis, and L. Tassiulas, "control of wireless networks with rechargeable batteries," IEEE Trans. Wireless Commun., vol. 9, no. 2, pp. 581-593, 2010.

[10] K. K. Kar, A. Krishnamurthy, and N. Jaggi, "Dynamic node activation in networks of rechargeable sensors," IEEE/ACM Trans. Networking, vol. 14, no. 1, pp. 15-26, Feb. 2006. 
[11] D. P. Bertsekas, Dynamic Programming and Optimal Control, 3rd ed. Massachusetts: Athena Scientific, 2007.

[12] J. G. Andrews, F. Baccelli, and R. K. Ganti, "A tractable approach to coverage and rate in cellular networks," IEEE Trans. Commun., under revision.

[13] M. Haenggi and R. K. Ganti, "Interference in Large Wireless Networks," Foundations and Trends in Networking, vol. 3, no. 2, pp. 127-248, 2008, available at http://www.nd.edu/ mhaenggi/pubs/now.pdf.

[14] N. Meuleau, K. E. Kim, L. P. Kaelbling, and A. R. Cassandra, "Solving POMDPs by searching the space of finite policies," in Proc. of the Fifteenth Conf. on Uncertainty in AI, 1999, pp. 417-426.

[15] J. Gozalvez, “Green radio technologies," IEEE Vehicular Technology Mag., vol. 5, no. 1, pp. 9-14, Mar. 2010.

[16] A. Gjendemsj, G. E. $\phi$ ien, and D. Gesbert, "Binary power control for multi-cell capacity maximization," in Proc. of IEEE 8th Workshop on Signal Processing Advances in Wireless Communications (SPAWC), Helsinki, Finland, 2007, pp. 1-5.

[17] N. Salodkar, “Online Algorithms for Delay Constrained Scheduling over a Fading Channel," Ph.D. dissertation, Indian Institute of Technology, May 2008.

[18] D. B. J. Abounadi and V. S. Borkar, "Learning algorithms for Markov decision processes with average cost," SIAM J. Control Optim., vol. 40, pp. 681-698, 2001.

[19] V. S. Borkar, "Stochastic approximation with two time scales," Systems and Control Lett., vol. 29, pp. 291-294, 1997.

[20] W. Szpankowski, "Stability conditions for some distributed systems: Buffered random access systems," Buffered Random Access Systems, Adv. Appl. Probab, vol. 26, pp. 498-515, 1993.

[21] J. Jeon and A. Ephremides, "The stability region of random multiple access under stochastic energy harvesting," in Proc. of IEEE International Symposium on Information Theory (ISIT), Saint-Petersburg, Russia, Aug. 2011, pp. 1796-1800.

[22] R. Rao and A. Ephremides, "On the stability of interacting queues in a multiple-access system," IEEE Trans. Inform. Theory, vol. 34, no. 5, pp. 918-930, Sept. 1988.

[23] 3rd Generation Partnership Project (3GPP), TR 25.996 v8.0.0, Spatial channel model for Multiple Input Multiple Output (MIMO) simulations. [Online]. Available: http://www.3gpp.org/technologies

[24] Y. Cui and V. K. N. Lau, "Distributive stochastic learning for delay-optimal OFDMA power and subband allocation," IEEE Trans. Signal Processing, vol. 58, no. 9, pp. 4848 - 4858, Sept. 2010.

[25] M. Haenggi, “On distance in uniformly random networks," IEEE Trans. Inform. Theory, vol. 51, no. 10, pp. 3584-3586, Oct. 2005.

[26] N. Jindal, J. G. Andrews, and S. Weber, "Multi-antenna communication in ad hoc networks: Achieving MIMO gains with SIMO transmission,” IEEE Trans. Commun., vol. 59, no. 2, pp. 529-540, Feb. 2011. 


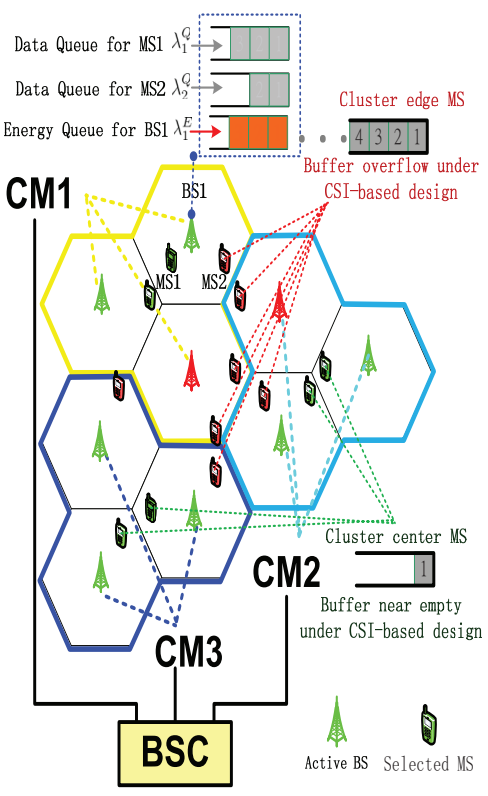

(a) System Model

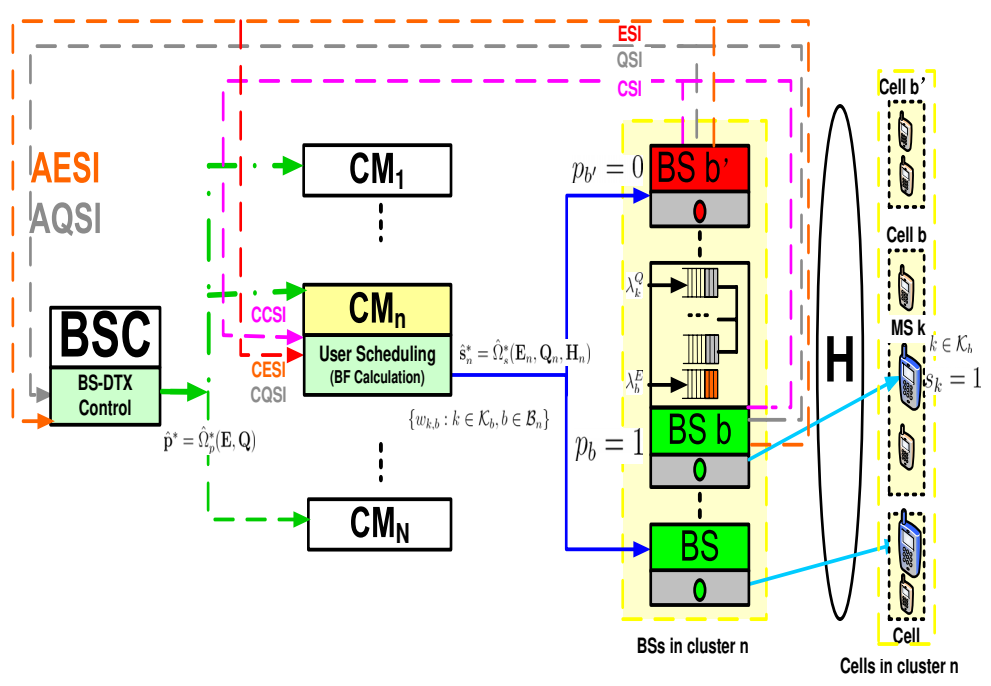

(b) Control Architecture

Fig. 1. System model and control architecture of the downlink coordinated MIMO systems. The dotted lines and solid lines on Fig 1 (b) denote the control path and data path, respectively.

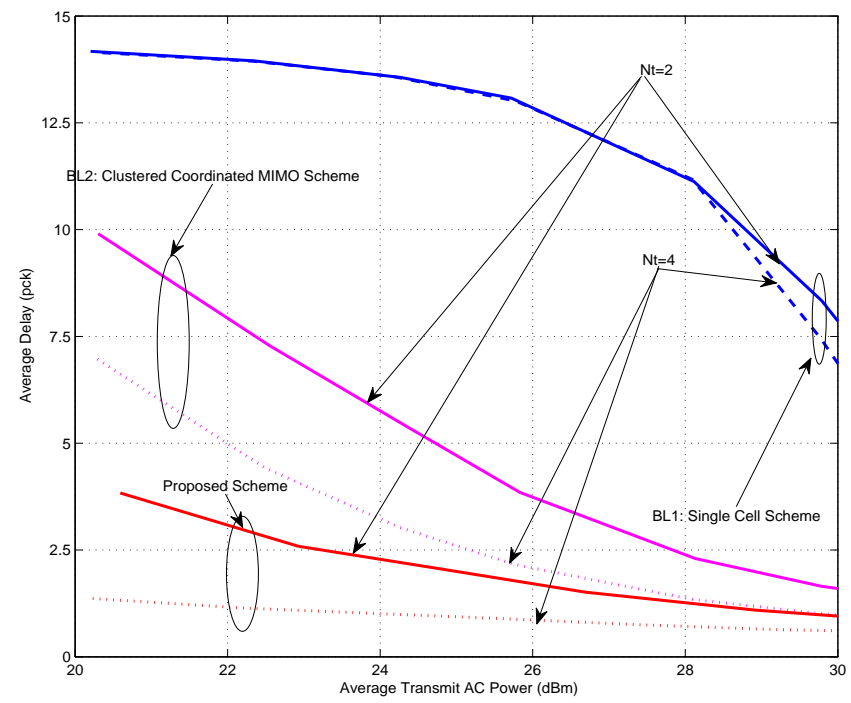

Fig. 2. Average delay versus average transmit grid power $(\mathrm{dBm})$ at $N_{t}=2$ and $N_{t}=4$. The average data arrival rate is $\lambda_{k}^{Q}=0.4 \mathrm{pck} / \mathrm{slot}$, the average renewable energy arrival rate is $\lambda_{b}^{E}=0.5 \mathrm{unit} / \mathrm{slot}$, and the energy storage size $N_{E}=4$ units for all $k \in \mathcal{K}$ and $b \in \mathcal{B}$. 


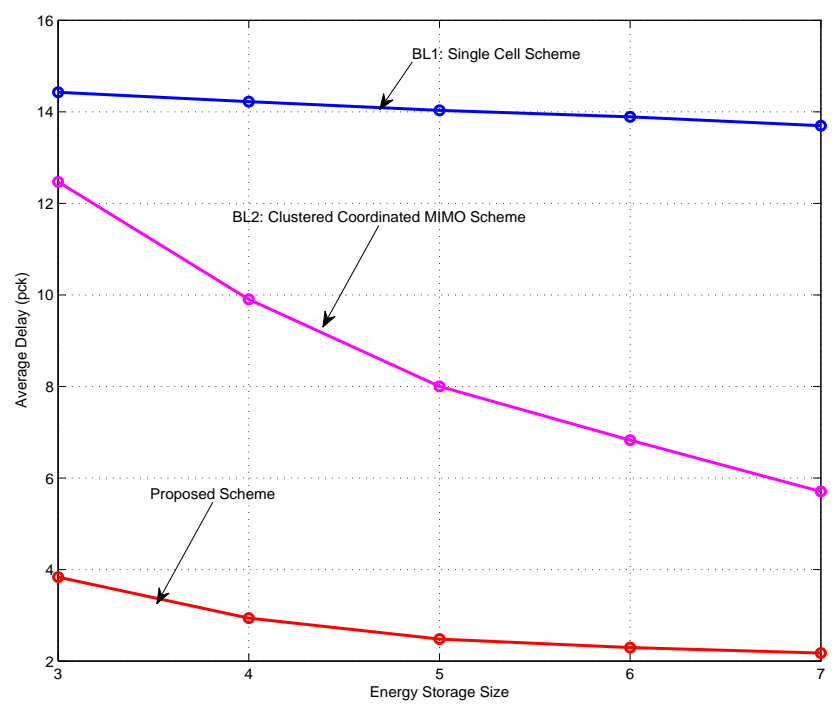

Fig. 3. Average delay versus energy storage size at average transmit grid power $15 \mathrm{dBm}$. The average data arrival rate is $\lambda_{k}^{Q}=0.4 \mathrm{pck} / \mathrm{slot}$ and the average renewable energy arrival rate is $\lambda_{b}^{E}=0.6$ unit/slot for all $k \in \mathcal{K}$ and $b \in \mathcal{B}$.

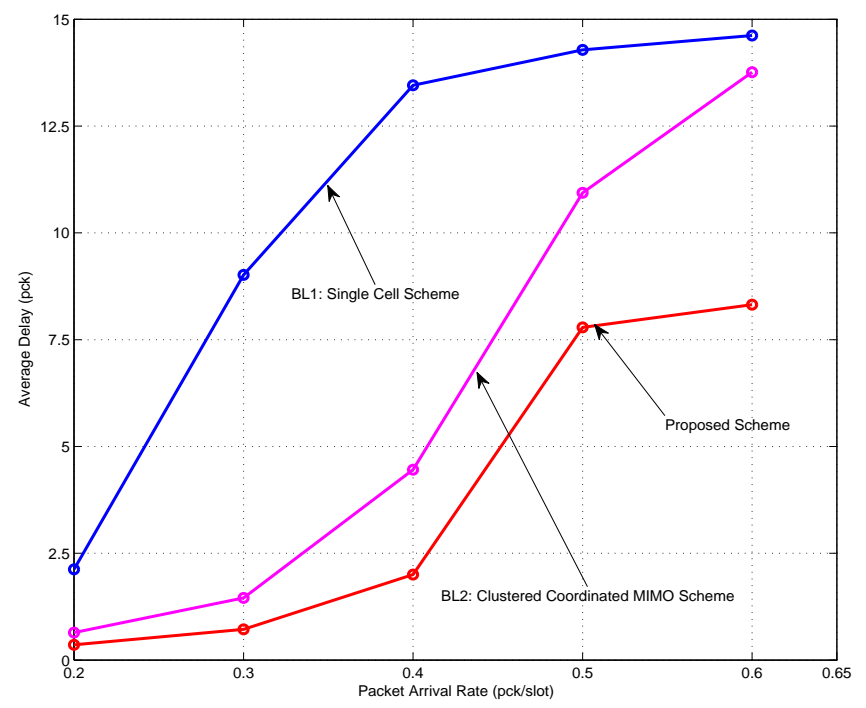

Fig. 4. Average delay versus average data arrival rate at average transmit grid power $25 \mathrm{dBm}$. The average renewable energy arrival rate is $\lambda_{b}^{E}=0.5$ unit/slot and the energy storage size $N_{E}=4$ for all $b \in \mathcal{B}$. 


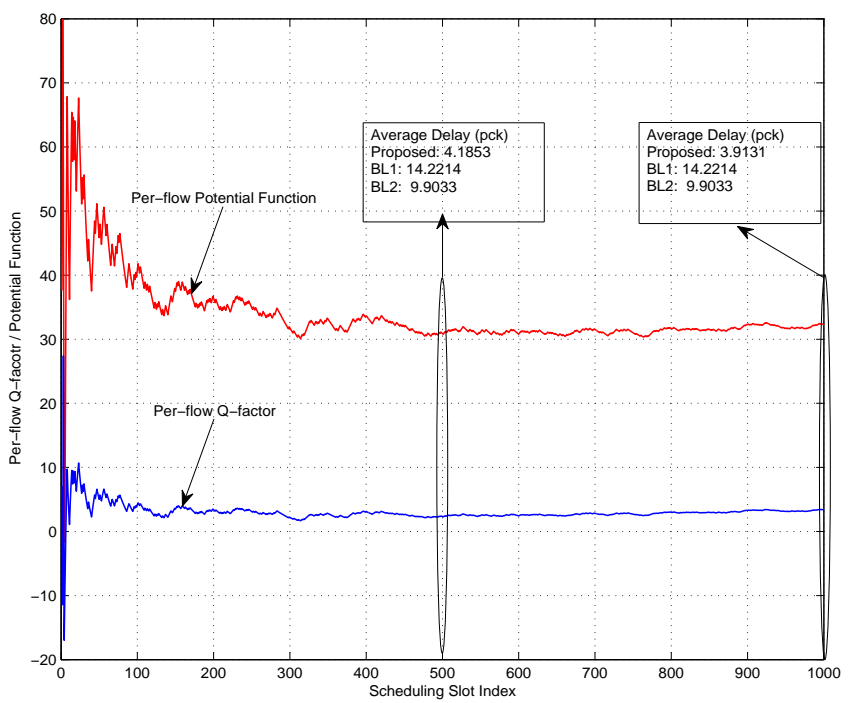

Fig. 5. Convergence property of the proposed distributed online learning algorithm at average transmit grid power 20 $\mathrm{dBm}$. The average data arrival rate is $\lambda_{k}^{Q}=0.4 \mathrm{pck} / \mathrm{slot}$, the average renewable energy arrival rate is $\lambda_{b}^{E}=0.5 \mathrm{unit} / \mathrm{slot}$, and the energy storage size $N_{E}=4$ for all $k \in \mathcal{K}$ and $b \in \mathcal{B}$. The figure illustrate the instantaneous per-flow post-decision potential function value $\hat{U}_{k}^{t}\left(\widetilde{E}_{b}, \widetilde{Q}_{k}\right)$ and the instantaneous per-flow Q-factor value $\hat{\mathbb{Q}}_{k}^{t}\left(E_{b}, Q_{k}, \mathbf{p}\right)$ respectively (during the online iterative updates in (22) and (21) versus instantaneous slot index, where $k=1, b=1, E_{b}=1, Q_{k}=1, \widetilde{E}_{b}=1$, $\widetilde{Q}_{k}=1$ and $\mathbf{p}=\left\{p_{b}=1: b \in \mathcal{B}\right\}$. The boxes indicate the average delay performance of various schemes at the two selected slot indices. 